\title{
Negative Saturation Approach for Non-Isothermal Compositional Two-Phase Flow Simulations
}

\author{
Hamidreza Salimi · Karl-Heinz Wolf • \\ Johannes Bruining
}

Received: 30 December 2010 / Accepted: 25 August 2011 / Published online: 23 September 2011 (C) Springer Science+Business Media B.V. 2011

\begin{abstract}
This article deals with developing a solution approach, called the non-isothermal negative saturation (NegSat) solution approach. The NegSat solution approach solves efficiently any non-isothermal compositional flow problem that involves phase disappearance, phase appearance, and phase transition. The advantage of the solution approach is that it circumvents using different equations for single-phase and two-phase regions and the ensuing unstable procedure. This paper shows that the NegSat solution approach can also be used for non-isothermal systems. The NegSat solution approach can be implemented efficiently in numerical simulators to tackle modeling issues for mixed $\mathrm{CO}_{2}$-water injection in geothermal reservoirs, thermal recovery processes, and for multicontact miscible and immiscible gas injection in oil reservoirs. We illustrate the approach by way of example to cold mixed $\mathrm{CO}_{2}-$ water injection in a 1D geothermal reservoir. The solution is compared with an analytical solution obtained with the wave-curve method (method of characteristics) and shows excellent agreement. A complete set of simulations is carried out, which identifies six bifurcations. The two main bifurcations are (1) when the most downstream compositional wave is replaced by a compositional shock and (2) when an extra Buckley-Leverett rarefaction appears. The plot of the useful energy (exergy) versus the $\mathrm{CO}_{2}$ storage capacity shows a $Z$-shape. The top horizontal part represents a branch of high exergy recovery/relatively lower storage capacity, whereas the bottom part represents a branch of lower exergy recovery/higher storage capacity.
\end{abstract}

Keywords Negative saturation approach · Non-isothermal compositional two-phase flow $\cdot \mathrm{CO}_{2}$ storage $\cdot$ Geothermal reservoirs 


\section{List of Symbols}

\section{Variables}

$C$ Heat capacity

$f \quad$ Fugacity

$F \quad$ Total molar variable

$g$ Gas

g Gravitational vector

$H$ Enthalpy

$k_{\mathrm{r}} \quad$ Relative permeability

$k_{\mathrm{T}} \quad$ Total thermal conductivity

K Absolute permeability tensor

$l \quad$ Liquid phase

$L \quad$ Liquid molar fraction

$n_{\mathrm{f}} \quad$ Number of degrees of freedom

$N_{\mathrm{c}} \quad$ Number of components

$N_{\mathrm{p}} \quad$ Number of phases

$P \quad$ Pressure

$P_{\mathrm{c}} \quad$ Capillary pressure

R Real

$S \quad$ Saturation

$\hat{S} \quad$ Extended saturation

$T$ Temperature

$u$ Total velocity

u Velocity vector

$V$ Gas molar fraction

$w \quad$ Water

$x \quad$ Mole fraction

$z \quad$ Overall mole fraction

$\mu \quad$ Viscosity

$\xi \quad$ Mass density

$\rho \quad$ Molar density

$\varphi \quad$ Porosity

$\Omega \quad$ Reservoir domain

\section{Subscripts}

$\begin{array}{ll}\mathrm{c} & \text { Compositional } \\ \mathrm{f} & \text { Freedom } \\ \mathrm{g} & \mathrm{CO}_{2} \text {-rich phase } \\ \mathrm{i} & \text { Component index } \\ \text { inj } & \text { Injection } \\ 1 & \text { Liquid phase } \\ \mathrm{p} & \text { Phase } \\ \mathrm{s} & \text { Solid reservoir rock } \\ \text { th } & \text { Thermal } \\ \alpha & \text { Phase index }\end{array}$




\section{Introduction}

Many problems in enhanced oil recovery (e.g., (im)miscible gas injection, chemical flooding, thermal recovery processes, and $\mathrm{CO}_{2}$ sequestration) involve transitions between single-phase and two-phase regions. Different systems of equations describe the behavior in the singlephase and in the two-phase regions (Lake 1989; Chen et al. 2006). Consequently, primary variables for each phase-state region will be different. A location in the reservoir can stay either in the two-phase (saturated) state or in the single-phase (undersaturated or oversaturated) state. Furthermore, from the $l$ th iteration to the $(l+1)$ th iteration in a Newton-Raphson (NR) procedure, the grid cell can stay in the same state or change to another phase state (Chen et al. 2006). Apart from the system of equations, also the thermodynamic conditions can vary in different phase states. Therefore, from the computational point of view, it is important to deal properly with the phase appearance and disappearance to obtain convergence of the NR procedure. The state of a reservoir can change from saturated to undersaturated (or oversaturated) or vice versa. Determination of a proper phase state during the state transition is called the bubble- or dew-point problem. In a bubble-point problem, the system can be in the single liquid phase or in the two-phase state, which requires solutions of a different set of equations. In a dew-point problem, the system can be in the single gas phase or in the two-phase state, which again requires solutions of a different set of equations. If the bubble-point/dew-point problem can be recognized promptly and reasonable unknowns can be selected for different phase states of a reservoir, convergence of the NR procedure can be enhanced.

There are four methods to deal with phase appearance and disappearance problems: (1) conventional models with switches to be used in grid cells where conversion of single-phase to two-phase or vice versa occurs (Bonnerot and Jamet 1981; Pruess 2004; Zhu et al. 2004; Chen et al. 2006; Bruining and Marchesin 2007); (2) a non-equilibrium source term in all the equations is used that drives the system toward equilibrium (Ben-Omran and Green 1978; Bruining and Van Duijn 2006); (3) compositional-space-parameterization approach (Voskov and Tchelepi 2009; Voskov 2010), which can be thought of as an extension of the conventional approach; and (4) the negative saturation method (Abadpour and Panfilov 2009; Panfilov and Rasoulzadeh 2010) that avoids the use of switches and separate equations.

Conventional algorithms require a cumbersome procedure to switch in a grid cell between single-phase and two-phase behavior. The main problem is that in a sequence of NR convergence steps, consecutive iterations may switch from single-phase to two-phase or vice versa. To circumvent the use of switches, it is also possible to formulate all equations with a source term that drives the system toward equilibrium. The disadvantage of this approach is that instead of component balances, we need to write the component balances in each phase, which almost doubles the number of equations. Moreover, the equations contain a usually highly nonlinear source term, which implicitly must be taken into account. The compositional-space-parameterization approach uses interpolated extended tie lines to connect the two-phase and single-phase regions, based on the method of characteristics. It uses an additional equation to calculate the density in single-phase regions. Recently, a new procedure called the negative saturation (NegSat) solution approach is introduced, which circumvents the use of cumbersome switches in isothermal reservoirs and does not require an additional equation. The NegSat solution approach was first introduced by Abadpour and Panfilov (2009) for isothermal flow without diffusion and by Panfilov and Rasoulzadeh (2010) for isothermal flow with diffusion. In this paper, we extend the NegSat solution approach to non-isothermal compositional flow simulations. Our motivation is that in many applications of interest, non-isothermal conditions prevail [e.g., in geothermal applications and thermal recovery (steam injection and in situ combustion)]. This requires that also the thermal parameters 
(e.g., enthalpies and heat capacities) must be defined in the single-phase regions in the same way as the non-thermal parameters (e.g., the relative permeabilities) in the isothermal systems. Without these extensions, the original NegSat solution approach is not capable of solving non-isothermal systems. Moreover, we have used full thermodynamic calculations to describe the phase behavior including the composition, temperature, and pressure dependence of the densities. This shows that the NegSat solution approach is not necessarily limited to the tie line approach and can be formulated in more general terms as is done here. We illustrate the non-isothermal NegSat solution approach by way of example to cold mixed $\mathrm{CO}_{2}$-water injection into a geothermal reservoir, because the non-isothermal characteristic of the flow plays a crucial role in geothermal reservoirs. A complete set of simulations can be used to obtain a first estimate of conditions for either optimal useful-energy recovery or optimal $\mathrm{CO}_{2}$ storage capacity.

The objective of this paper is to assess the applicability of the non-isothermal NegSat solution approach. Therefore, the procedure must be capable to model the phase disappearance/appearance as well as the phase transition between subcooled and supercritical behavior. By subcooled, we mean below the critical temperature of $\mathrm{CO}_{2}$ but above the critical pressure. Supercritical means both above the critical temperature and above the critical pressure. Subcritical $\mathrm{CO}_{2}$ (i.e., below the critical pressure and temperature) is not considered in this paper. Note that the phase states involved are liquid, gas or supercritical.

No attempt has yet been made to model the phase appearance and disappearance for problems that involve both complex thermodynamics and non-isothermal flow, and this is the innovative aspect of this paper. We also introduce a plot of the useful-energy extraction (energy that can be exploited) and the storage capacity, which can be used to locate optimal in situ conditions.

The paper is organized as follows. First, we write the systems of equations for single-phase and two-phase regions. Then, we briefly explain the standard solution technique to deal with problems that involve phase appearances and disappearances. Subsequently, we derive the NegSat solution approach for non-isothermal compositional flow. Here, the non-isothermal NegSat solution approach replaces a single-phase multi-component fluid by an equivalent fictitious two-phase fluid with specific properties. Furthermore, we illustrate full details of the derivation of the NegSat solution approach for non-isothermal compositional two-phase flow simulations. In addition, we demonstrate the approach with some examples and solutions, which are compared with analytical solutions obtained with the wave-curve method, an extension of the fractional-flow theory. We also give a full set of simulation results that shows the possible bifurcations of cold mixed $\mathrm{CO}_{2}$-water injection into a geothermal reservoir. $\mathrm{A}$ plot of the results in a useful-energy/storage-capacity diagram is described in detail. Finally, we summarize our findings in the conclusion section.

\section{Model Equations for Non-Isothermal Flow}

We consider a situation in which the components (e.g., $\mathrm{CO}_{2}$ and $\mathrm{H}_{2} \mathrm{O}$ ) form at most two phases (e.g., gas and aqueous). For reasons of concise notation, the subcooled/supercritical $\mathrm{CO}_{2}$-rich and the aqueous phase are denoted by gas and liquid, respectively. However as we have derived the equations for $N_{\mathrm{c}}$ components, the theory is valid for $N_{\mathrm{c}}$ components in two phases. The governing equations for non-isothermal compositional two-phase (liquid/gas) flow are given by the species-balance equations, Darcy's law, the energy conservation equation, and the thermodynamic equilibrium between the phases. A possible salinity of the aqueous phase is not considered. 


\subsection{Two-Phase Equations}

Let $\varphi$ and $\mathbf{K}$ denote the porosity and permeability of a geothermal reservoir $\Omega \subset \mathrm{R}^{3}$, and let $S_{\alpha}, \mu_{\alpha}, P_{\alpha}, \mathbf{u}_{\alpha}, k_{\mathrm{r} \alpha}$ be the saturation, viscosity, pressure, volumetric velocity, and relative permeability of phase $\alpha, \alpha=l, g$, respectively. The molar density of phase $\alpha$ is given by

$$
\rho_{\alpha}=\sum_{i=1}^{N_{\mathrm{c}}} \rho_{i \alpha}, \quad \alpha=l, g,
$$

where $\rho_{i \alpha}$ represents the molar density of component $i$ in phase $\alpha$. The mole fraction of component $i$ in phase $\alpha$ is then defined by

$$
x_{i \alpha}=\frac{\rho_{i \alpha}}{\rho_{\alpha}}, \quad i=1,2, \ldots, N_{\mathrm{c}}, \quad \alpha=l, g .
$$

The total mass is conserved for each component

$$
\frac{\partial}{\partial t} \varphi \sum_{\alpha=l}^{g} x_{i \alpha} \rho_{\alpha} S_{\alpha}+\nabla \cdot \sum_{\alpha=l}^{g} x_{i \alpha} \rho_{\alpha} \mathbf{u}_{\alpha}=0, \quad i=1,2, \ldots, N_{\mathrm{c}},
$$

where the volumetric velocity $\mathbf{u}_{\alpha}$ is given by Darcy's law, i.e.,

$$
\mathbf{u}_{\alpha}=-\frac{k_{\mathrm{r} \alpha}}{\mu_{\alpha}} \mathbf{K}\left(\nabla P_{\alpha}+\xi_{\alpha} \mathbf{g}\right), \quad \alpha=l, g .
$$

Here, $\xi_{\alpha}$ is the mass density of phase $\alpha$ and $\mathbf{g}$ is the gravitational vector. In Eq. 4 , the vertical direction is upward. The difference in phase pressures is related by the capillary pressure

$$
P_{\mathrm{g}}-P_{1}=P_{\mathrm{c}}\left(S_{1}\right) \text {. }
$$

As usual, if the kinetic and potential energy and friction terms in the energy conservation equation are neglected, the energy conservation equation takes the form

$$
\frac{\partial}{\partial t}\left(\varphi \sum_{\alpha=l}^{g} \rho_{\alpha} S_{\alpha} H_{\alpha}+(1-\varphi) \rho_{\mathrm{s}} C_{\mathrm{s}} T\right)+\nabla \cdot \sum_{\alpha=l}^{g} \rho_{\alpha} \mathbf{u}_{\alpha} H_{\alpha}-\nabla \cdot\left(k_{\mathrm{T}} \nabla T\right)=0,
$$

where we further neglect pressure-volume work by letting the enthalpies equal internal energies (Lake 1989). In Eq. 6, $T$ denotes the temperature, $H_{\alpha}$ is the specific enthalpy of phase $\alpha$ (per unit mass), $\rho_{\mathrm{s}}$ and $C_{\mathrm{S}}$ are the density and the specific heat capacity of the solid porous rock and $k_{\mathrm{T}}$ represents the total thermal conductivity. In Eq. 6, the specific enthalpy $H_{\alpha}$ of phase $\alpha$ can be computed from

$$
H_{\alpha}=C_{\mathrm{p} \alpha} T,
$$

where $C_{\mathrm{p} \alpha}$ denotes the heat capacity of phase $\alpha$ at constant pressure. Here, we disregard the energy of mixing.

In addition to the differential equations (Eqs. 3, 4, and 6), the compositions are constrained by

$$
\sum_{i=1}^{N_{\mathrm{c}}} x_{i \alpha}=1, \quad \alpha=l, g
$$


The local thermodynamic equilibrium implies the equality of the fugacities of each component in the two phases. Therefore, the equilibrium relation describing the mass distribution of components into the phases is given by

$$
f_{i 1}\left(T, P_{1}, x_{j 1 ; j=1,2, \ldots, N_{\mathrm{c}}-1}\right)=f_{i \mathrm{~g}}\left(T, P_{\mathrm{g}}, x_{j \mathrm{~g} ; j=1,2, \ldots, N_{\mathrm{c}}-1}\right), \quad i=1,2, \ldots, N_{\mathrm{c}},
$$

where $f_{i \alpha}$ is the fugacity of the $i$ th component in phase $\alpha$. In addition, we use the total molar variable $F$ of the $\mathrm{CO}_{2}$-water system (Chen et al. 2006)

$$
F=\rho_{\mathrm{l}} S_{1}+\rho_{\mathrm{g}} S_{\mathrm{g}},
$$

and the molar fractions of liquid $(L)$ and gas $(V)$ in this system,

$$
L=\frac{\rho_{\mathrm{l}} S_{1}}{F}, \quad V=\frac{\rho_{\mathrm{g}} S_{\mathrm{g}}}{F} .
$$

Note that

$$
L+V=1
$$

Then, the overall mole fraction of the components in the system can be written as

$$
z_{i}=(1-V) x_{i 1}+V x_{i \mathrm{~g}}, \quad i=1,2, \ldots, N_{\mathrm{c}} .
$$

Evidently, the sum of the overall mole fractions must be one, which is found by summing Eq. 11 over the components and by using Eqs. 7, 9, and 10

$$
\sum_{i=1}^{N_{\mathrm{c}}} z_{i}=1 .
$$

Then, we finally find

$$
x_{i 1} \rho_{\mathrm{l}} S_{\mathrm{l}}+x_{i \mathrm{~g}} \rho_{\mathrm{g}} S_{\mathrm{g}}=F z_{i}, \quad i=1,2, \ldots, N_{\mathrm{c}} .
$$

The two-phase region includes the situation that one of the phases is immobile but we do not consider a solid phase.

\subsection{Single-Phase Equations}

The equations used to model the non-isothermal transport of multicomponents in a single fluid phase are similar to Eqs. 3, 4, and 6, i.e.,

$$
\frac{\partial}{\partial t}\left(\varphi z_{i} \rho_{\alpha}\right)+\nabla .\left(z_{i} \rho_{\alpha} \mathbf{u}_{\alpha}\right)=0, \quad i=1,2, \ldots, N_{\mathrm{c}}, \quad \alpha=l, \text { or } \alpha=g,
$$

where, again, we disregard diffusion/dispersion effects. The relation for Darcy's volumetric velocity is the same as Eq. 4 for two-phase regions, except that the relative permeability becomes one for single-phase regions. The constraint for the single-phase compositions also remains the same as in Eq. 12. The energy conservation equation for single-phase can be approximated by

$$
\frac{\partial}{\partial t}\left(\varphi \rho_{\alpha} H_{\alpha}+(1-\varphi) \rho_{\mathrm{s}} C_{\mathrm{s}} T\right)+\nabla \cdot\left(\rho_{\alpha} \mathbf{u}_{\alpha} H_{\alpha}\right)-\nabla \cdot\left(k_{\mathrm{T}} \nabla T\right)=0 .
$$




\section{Standard Solution Techniques}

The simultaneous solution technique has to be used in a non-isothermal flow problem because of strong nonlinearity and coupling in the governing equations. In addition, pressure and temperature vary considerably, and mass transfer and energy transfer occur frequently between the phases. Indeed, the governing equations for non-isothermal compositional flow form a strongly coupled system of time-dependent, nonlinear differential equations, and algebraic constraints. Although the number of equations and the number of dependent variables are the same, the system of equations can be written in terms of primary variables, and other variables can be expressed as functions of them. These primary variables must be carefully chosen, so that they describe the main physical properties while the constraints are preserved, and the nonlinearity and coupling between the equations are weakened. Thus, efficient numerical methods for the solution of the resulting system can be developed.

Both for non-isothermal and for isothermal compositional flow, there is usually a connected sequence of single-phase and two-phase flow regions. This is also the case for cold mixed $\mathrm{CO}_{2}$-water injection into a geothermal reservoir. As shown above, different systems of equations apply for single-phase and two-phase regions. The standard solution technique treats the single-phase region and the two-phase region separately by solving different systems of equations for each phase-state region. As a result, the choice of the primary unknowns depends on the phase state of the reservoir.

\subsection{Phase States}

For two-phase flow, the overall mole fraction of component $i$ is bounded between $\left(x_{i 1}\right)$ and $\left(x_{i \mathrm{~g}}\right)$. For the $\mathrm{CO}_{2}$-water system, $\left(x_{\mathrm{CO}_{2}, \mathrm{l}}\right)<\left(x_{\mathrm{CO}_{2}, \mathrm{~g}}\right)$ holds. Therefore, if the overall $\mathrm{CO}_{2}$ mole fraction $\left(z_{\mathrm{CO}_{2}}\right)$ is smaller than the $\mathrm{CO}_{2}$ mole fraction in the aqueous phase $\left(x_{\mathrm{CO}_{2}, 1}\right)$ obtained from the flash calculation (i.e., $z_{\mathrm{CO}_{2}}<x_{\mathrm{CO}_{2}, 1}$ ), there is only single-phase aqueous flow and the reservoir is in the undersaturated state. In this case, all $\mathrm{CO}_{2}$ dissolves into the aqueous phase (i.e., $S_{\mathrm{g}}=0$ ). In the same way, if the overall $\mathrm{CO}_{2}$ mole fraction is larger than the $\mathrm{CO}_{2}$ mole fraction in the $\mathrm{CO}_{2}$-rich phase (either subcooled, supercritical, or gas) obtained from the flash calculation (i.e., $z_{\mathrm{CO}_{2}}>x_{\mathrm{CO}_{2}, \mathrm{~g}}$ ), then, there is a $\mathrm{CO}_{2}$-rich single-phase and the reservoir is referred to as being in the oversaturated state. In this case, all water dissolves into the $\mathrm{CO}_{2}$-rich phase (i.e., $S_{1}=0$ ). Finally, if $x_{\mathrm{CO}_{2}, 1}<z_{\mathrm{CO}_{2}}<x_{\mathrm{CO}_{2}, \mathrm{~g}}$, there are two phases and the reservoir is said to be in the saturated state. Because of the frequent change in reservoir temperature and pressure, the flash calculations vary. Consequently, transitions from a single-phase state to a two-phase state and vice versa can occur.

\subsection{Choice of Primary Variables}

Because the choice of the primary unknowns is dependent on the reservoir phase state, we separate the discussion of the saturated state from that of the undersaturated/oversaturated state.

\subsubsection{Saturated State}

As discussed earlier, if two phases coexist, a reservoir is in the saturated state. In this state, there is a free $\mathrm{CO}_{2}$-rich (referred to as gas) phase. Pressure, temperature, and compositions are related by the local thermodynamic equilibrium relations. Thus, a combination of them can be used as primary variables. For cold mixed $\mathrm{CO}_{2}$-water injection into a geothermal 
reservoir, the primary unknowns are chosen as $\left(P, S_{\mathrm{g}}, T, z_{1}, z_{2}, \ldots, z_{N c-2}\right)$, where $P=P_{\mathrm{g}}$. This follows from Gibbs' phase rule where the number of degrees of freedom $n_{\mathrm{f}}=N_{\mathrm{c}}-$ $N_{\mathrm{p}}+(T, P)$. The saturation is an additional primary variable. Therefore, the number of primary variables for a two-phase system $\left(N_{\mathrm{p}}=2\right)$ is $N_{\mathrm{c}}+1$. The system of differential equations is composed of the $N_{\mathrm{c}}$ component mass conservation equations and the energy conservation equation. Recall that the capillary pressure $P_{\mathrm{c}}$ and relative permeabilities $k_{\mathrm{r} \alpha}$ are known functions of saturation and that the viscosity $\mu_{\alpha}$ and molar densities $\rho_{\alpha}$ are functions of their respective phase pressure, compositions, and temperature.

\subsubsection{Undersaturated/Oversaturated State}

When all $\mathrm{CO}_{2}$ dissolves into the aqueous phase (i.e., $S_{\mathrm{g}}=0$ ) or all the water dissolves into the $\mathrm{CO}_{2}$-rich phase (i.e., $S_{1}=0$ ), the reservoir is in the undersaturated state or in the oversaturated state, respectively. In the undersaturated/oversaturated state, $\left(P, T, z_{1}, z_{2}, \ldots, z_{N c-1}\right)$ are chosen as the primary variables. This follows from Gibbs' phase rule where the number of degrees of freedom is $n_{\mathrm{f}}=N_{\mathrm{c}}-N_{\mathrm{p}}+(T, P)$. The saturation is now given (i.e., is one or zero). Therefore, the number of primary variables for a single-phase system $\left(N_{\mathrm{p}}=1\right)$ is again $N_{\mathrm{c}}+1$. This is equal to the number of equations (i.e., $N_{\mathrm{c}}$ component balances and one energy equation).

As discussed earlier, during non-isothermal compositional flow, it is possible to have a sequence of different phase-state regions at different times for the entire reservoir. Determination of a proper phase state during the state transition is the bubble-point/dew-point problem. Because the standard solution technique solves different equations for each phasestate region, it needs to deal with different primary variables in each region. Moreover, the standard solution technique must use a phase-state switch to deal with the transition of phase states in the reservoir. Therefore, it is very important to deal properly with the bubble-point/ dew-point problem to control convergence of the NR procedure. The phase state of a reservoir can change from undersaturated (or oversaturated) to saturated or vice versa. If the bubble (or dew) point can be promptly recognized and reasonable unknowns can be selected for different phase states of a reservoir, then in the standard solution technique, the rate of convergence of the NR procedure can be better monitored and enhanced.

To handle the bubble-point/dew-point problem properly in the standard solution technique, the method described by Chen et al. (2006) can be adopted. In essence, this standard solution technique requires at each grid cell:

- A stability test to check whether the mixture can form two phases at the current conditions, if it was originally in a single-phase condition or vice versa (Michelsen 1982a);

- A flash computation and a switch of the status of the cell to two-phase, if it can form two phases (Michelsen 1982b);

- An implicit flash computation where thermodynamic constraints converged simultaneously with the transport equations in the same NR loop, if the cell is already in the two-phase state.

The main drawbacks of the standard solution technique are that the required tests are timeconsuming and that the switches may be ambiguous. By this, we mean that the tests propose simultaneously a switch from the single-phase state to the two-phase state and vice versa.

To overcome these challenges associated with the standard solution technique, a new approach, called the negative saturation approach, was proposed (Abadpour and Panfilov 2009; Panfilov and Rasoulzadeh 2010) to derive a unique system of two-phase flow equations for isothermal compositional flow. In this paper, we extend the negative saturation 
approach to non-isothermal compositional two-phase flow and obtain the non-isothermal negative saturation approach. This requires that also the thermal parameters (e.g., enthalpies and heat capacities) must be defined in the single-phase regions in the same way as the non-thermal parameters (e.g., the relative permeabilities) in the isothermal systems. Without these extensions, the original NegSat solution approach is not capable of solving non-isothermal systems. Moreover, we have used full thermodynamic calculations to describe the phase behavior including the composition, temperature, and pressure dependence of the densities. This shows that the NegSat solution approach is not necessarily limited to the tie line approach and can be formulated in more general terms as is done here. Below, we give full derivations of this approach.

\section{Non-Isothermal Negative Saturation Approach}

We formulate the NegSat solution approach for non-isothermal compositional two-phase flow. To be able to have a uniform system of equations for the entire reservoir that could properly deal with different phase states of the reservoir without changing the primary variables and constraint conditions, we need to know beforehand how many phases could coexist at most. For cold mixed $\mathrm{CO}_{2}$-water injection into a geothermal reservoir, two phases could coexist at most (viz., a $\mathrm{CO}_{2}$-rich phase and a water-rich phase). Therefore, we start with replacing the equations for single-phase regions (i.e., oversaturated and undersaturated) with the equations for equivalent fictitious two-phase regions with specific properties. We use the principle of equivalence to derive the specific properties. We use a "hat" on the dependent variables to indicate that they can be used both in single-phase and two-phase flow in the transport, momentum (Darcy's law), and energy equations. They read

$$
\begin{aligned}
& \frac{\partial}{\partial t}\left(\varphi\left(\hat{x}_{\mathrm{g}} \hat{\rho}_{\mathrm{g}} \hat{S}_{\mathrm{g}}+\hat{x}_{i 1} \hat{\rho}_{\mathrm{l}}\left(1-\hat{S}_{\mathrm{g}}\right)\right)\right)+\nabla \cdot\left(\hat{x}_{\mathrm{g}} \hat{\rho}_{\mathrm{g}} \hat{\mathbf{u}}_{\mathrm{g}}+\hat{x}_{i 1} \hat{\rho}_{\mathrm{l}} \hat{\mathbf{u}}_{\mathrm{l}}\right)=0, \quad i=1,2, \ldots, N_{\mathrm{c}}, \\
& z_{i}=\frac{\hat{x}_{\mathrm{g}} \hat{\rho}_{\mathrm{g}} \hat{S}_{\mathrm{g}}+\hat{x}_{i 1} \hat{\rho}_{\mathrm{l}}\left(1-\hat{S}_{\mathrm{g}}\right)}{\hat{\rho}_{\mathrm{g}} \hat{S}_{\mathrm{g}}+\hat{\rho}_{\mathrm{l}}\left(1-\hat{S}_{\mathrm{g}}\right)}, \quad i=1,2, \ldots, N_{\mathrm{c}} \\
& \hat{\mathbf{u}}_{\mathrm{g}}=-\frac{\hat{k}_{\mathrm{rg}}}{\hat{\mu}_{\mathrm{g}}} \mathbf{K}\left(\nabla \hat{P}_{\mathrm{g}}+\hat{\xi}_{\mathrm{g}} \mathrm{g}\right), \quad \text { and } \quad \hat{\mathbf{u}}_{\mathrm{l}}=-\frac{\hat{k}_{\mathrm{rl}}}{\hat{\mu}_{\mathrm{l}}} \mathbf{K}\left(\nabla \hat{P}_{1}+\hat{\xi}_{\mathrm{g}} \mathrm{g}\right) \\
& \frac{\partial}{\partial t}\left(\varphi T\left(\hat{\rho}_{\mathrm{g}} \hat{S}_{\mathrm{g}} \hat{C}_{\mathrm{pg}}+\hat{\rho}_{\mathrm{l}}\left(1-\hat{S}_{\mathrm{g}}\right) \hat{C}_{\mathrm{pl}}\right)+(1-\varphi) \rho_{\mathrm{s}} C_{\mathrm{s}} T\right) \\
& \quad+\nabla \cdot\left(T\left(\hat{\rho}_{\mathrm{g}} \hat{\mathbf{u}}_{\mathrm{g}} \hat{C}_{\mathrm{pg}}+\hat{\rho}_{\mathrm{l}} \hat{\mathbf{u}}_{\mathrm{l}} \hat{C}_{\mathrm{pl}}\right)\right)-\nabla \cdot\left(k_{\mathrm{T}} \nabla T\right)=0
\end{aligned}
$$

We do not use a hat on the temperature, because its definition does not depend on the phase state. Because the equations are the same as the two-phase transport equations, we call Eqs. 16, 17, 18, 19 the fictitious two-phase equations.

\subsection{Specific Properties}

For two-phase regions, the fictitious two-phase flow equations exactly remain the same as the classical two-phase flow equations. The principle of equivalence makes it possible to define expressions for all symbols with a hat, so that they also describe the single-phase flow behavior. If we use these expressions, we convert two-phase flow equations to single-phase 
flow equations. Undersaturated liquid is distinguished from oversaturated gas, because there are different specific properties for each phase state.

\subsubsection{Undersaturated State}

The principle of equivalence implies that firstly the undersaturated phase molar density should be equal to the total molar density of the fictitious two phases, i.e.,

$$
\rho_{\mathrm{l}}=\hat{\rho}_{\mathrm{g}} \hat{S}_{\mathrm{g}}+\hat{\rho}_{\mathrm{l}}\left(1-\hat{S}_{\mathrm{g}}\right) .
$$

Consequently, for any equivalent gas saturation $\hat{S}_{\mathrm{g}}$, we must have that

$$
\hat{\rho}_{\mathrm{g}}=\hat{\rho}_{1}=\rho_{\mathrm{l}} \text {. }
$$

Secondly, the overall concentration of component $i$ in the undersaturated phase must be equal to that in the fictitious two phases, i.e.,

$$
z_{i}=\hat{x}_{i \mathrm{~g}} \hat{S}_{\mathrm{g}}+\hat{x}_{i 1}\left(1-\hat{S}_{\mathrm{g}}\right), \quad i=1,2, \ldots, N_{\mathrm{c}},
$$

where we have already used Eq. 21 to derive Eq. 22.

Thirdly, the undersaturated phase flux must be equated to the total flux of the fictitious two phases. Using Eqs. 18, 21, and 22, this flux equality reads

$$
\begin{aligned}
& \left(\hat{x}_{i \mathrm{~g}} \hat{S}_{\mathrm{g}}+\hat{x}_{i 1}\left(1-\hat{S}_{\mathrm{g}}\right)\right) \frac{1}{\mu_{\mathrm{l}}}\left(\nabla P_{1}+\xi_{1} \mathbf{g}\right) \\
& \quad=\left(\hat{x}_{i \mathrm{~g}} \frac{\hat{k}_{\mathrm{rg}}}{\hat{\mu}_{\mathrm{g}}}\left(\nabla \hat{P}_{\mathrm{g}}+\hat{\xi}_{\mathrm{g}} \mathbf{g}\right)+\hat{x}_{i 1} \frac{\hat{k}_{\mathrm{rl}}}{\hat{\mu}_{1}}\left(\nabla \hat{P}_{1}+\hat{\xi}_{1} \mathbf{g}\right)\right), \quad i=1,2, \ldots, N_{\mathrm{c}}
\end{aligned}
$$

where the absolute permeability tensor $\mathbf{K}$ is dropped at both sides of Eq. 23. From Eq. 23, we could derive specific properties for viscosity, relative permeabilities, pressures, and mass densities. To do so, it is enough to expand the left side of Eq. 23 and compare it with the right side of Eq. 23; we obtain

$$
\begin{aligned}
& \hat{\mu}_{\mathrm{g}}=\hat{\mu}_{1}=\mu_{1}, \\
& \hat{P}_{\mathrm{g}}=\hat{P}_{1}=P_{1}, \\
& \hat{\xi}_{\mathrm{g}}=\hat{\xi}_{1}=\xi_{1}, \\
& \hat{k}_{\mathrm{rg}}=\hat{S}_{\mathrm{g}}, \quad \hat{k}_{\mathrm{rl}}=\left(1-\hat{S}_{\mathrm{g}}\right),
\end{aligned}
$$

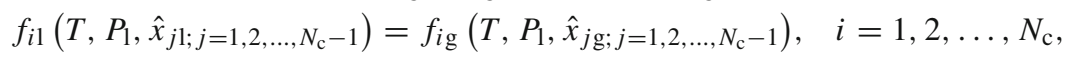

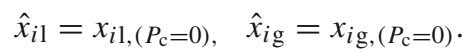

Equation 25 indicates that the capillary pressure $P_{\mathrm{c}}$ becomes zero for the undersaturated phase. Note that in Eqs. 22 and 23, the mole fraction of component $i$ in the fictitious two phases are obtained from Eq. 28 (i.e., the flash calculation based on local thermodynamic equilibrium at zero $P_{\mathrm{c}}$ ). The flash calculation for the undersaturated phase is the same as for the negative flash calculation (Whitson and Michelsen 1989).

Finally, the energy conservation equation for the undersaturated phase (Eq. 15) must be equivalent to that for the fictitious two phases (Eq. 19). If we use Eq. 21, and Eqs. 24-27, we obtain

$$
\left(\hat{S}_{\mathrm{g}} \hat{C}_{\mathrm{pg}}+\left(1-\hat{S}_{\mathrm{g}}\right) \hat{C}_{\mathrm{pl}}\right)=C_{\mathrm{pl}}, \Rightarrow \hat{C}_{\mathrm{pg}}=\hat{C}_{\mathrm{pl}}=C_{\mathrm{pl}}
$$


To our knowledge, this result and the equivalent result for the oversaturated state have not been derived before. The derivations of the specific properties for the undersaturated phase are now complete. The saturation of the equivalent gas $\hat{S}_{\mathrm{g}}$ is called the extended gas saturation. If we rearrange Eq. 22, we derive for the extended saturation

$$
\hat{S}_{\mathrm{g}}=\frac{z_{i}-\hat{x}_{i 1}}{\hat{x}_{i \mathrm{~g}}-\hat{x}_{i 1}}, \quad i=1,2, \ldots, N_{\mathrm{c}} .
$$

The extended gas saturation for the undersaturated state is negative (i.e., $\hat{S}_{\mathrm{g}}<0$ ). The reason is as follows. For light components (i.e., $x_{i \mathrm{~g}} / x_{i 1}>1$ ), the phase state of a system, for a given temperature $T$ and pressure $P$, is undersaturated when $z_{i}<x_{i 1}$ (see Eq. 11). Therefore, the numerator of Eq. 30 is negative, and the denominator of Eq. 30 is positive. As a result, the extended gas saturation $\hat{S}_{\mathrm{g}}$ becomes negative. Also, for heavy components in a two-phase mixture (i.e., $x_{i \mathrm{~g}} / x_{i 1}<1$ ), the phase state of a system, for a given temperature $T$ and pressure $P$, is undersaturated when $z_{i}>x_{i 1}$. Hence, the numerator of Eq. 30 is now positive and the denominator of Eq. 30 is now negative. As a result, the extended gas saturation (Eq. 30) again becomes negative. When the extended saturation is zero, the phase state of a system is at the bubble point.

\subsubsection{Oversaturated State}

Analogous to the undersaturated state, the principal of equivalence implies that from the physical point of view, the fictitious two phases should have the following properties to become the same as the oversaturated phase:

$$
\begin{aligned}
& \hat{\rho}_{\mathrm{g}}=\hat{\rho}_{\mathrm{l}}=\rho_{\mathrm{g}}, \\
& \hat{\mu}_{\mathrm{g}}=\hat{\mu}_{1}=\mu_{\mathrm{g}} \text {, } \\
& \hat{P}_{\mathrm{g}}=\hat{P}_{1}=P_{\mathrm{g}} \text {, } \\
& \hat{\xi}_{\mathrm{g}}=\hat{\xi}_{1}=\xi_{\mathrm{g}}, \\
& \hat{k}_{\mathrm{rg}}=\hat{S}_{\mathrm{g}}, \quad \hat{k}_{\mathrm{rl}}=\left(1-\hat{S}_{\mathrm{g}}\right), \\
& \left(\hat{S}_{\mathrm{g}} \hat{C}_{\mathrm{pg}}+\left(1-\hat{S}_{\mathrm{g}}\right) \hat{C}_{\mathrm{pl}}\right)=C_{\mathrm{pg}}, \Rightarrow \hat{C}_{\mathrm{pg}}=\hat{C}_{\mathrm{pl}}=C_{\mathrm{pg}},
\end{aligned}
$$

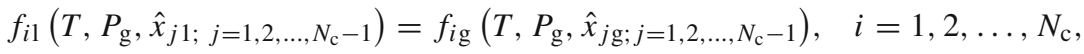

$$
\begin{aligned}
& \hat{x}_{i 1}=x_{i 1,\left(P_{\mathrm{c}}=0\right)}, \quad \hat{x}_{i \mathrm{~g}}=x_{i \mathrm{~g},\left(P_{\mathrm{c}}=0\right)} \text {. }
\end{aligned}
$$

Note that Eq. 37 is the same flash calculation as Eq. 8, except that it is now at zero capillary pressure (i.e., $P_{\mathrm{c}}=0$ ). At this stage, we still distinguish between the fugacity of the liquid and gas phase. Whether we are in the oversaturated state is determined as follows. For the oversaturated state, the extended gas saturation is positive and larger than unity (i.e., $\hat{S}_{\mathrm{g}}>1$ ). The reason is that for light components in a mixture (i.e., $x_{i \mathrm{~g}} / x_{i 1}>1$ ), the phase state of a system, for a given temperature $T$ and pressure $P$, is oversaturated when $z_{i}>x_{i \mathrm{~g}}$. Therefore, the numerator of Eq. 30 is positive and is larger than the positive denominator of Eq. 30. As a result, the extended gas saturation $\hat{S}_{\mathrm{g}}$ becomes larger than unity. In the same way, for heavy components in a two-phase mixture (i.e., $x_{i \mathrm{~g}} / x_{i 1}<1$ ), the phase state of a system, at a given temperature $T$ and pressure $P$, is oversaturated when $z_{i}<x_{i \mathrm{~g}}$. Hence, the numerator of Eq. 30 is now negative and is smaller than the negative denominator of Eq. 30. Therefore, the extended gas saturation (Eq. 30) is again larger than unity. When the extended gas saturation is one, the phase state of a system is at the dew point. 
In summary, when the extended gas saturation is between zero and one, it is the same as the actual gas saturation. If the extended gas saturation is above one, we have a single gaseous phase and the actual gaseous saturation is one. If the extended gas saturation is below zero, we have a single liquid phase and the actual gas saturation is zero.

As mentioned previously, the advantage of the negative-saturation approach formulation is to avoid variable substitution when the number of phases changes. In both single- and two-phase regions, we continue to keep the same system of equations as well as primary unknowns. In single-phase regions, for known $\hat{S}_{\mathrm{g}}, \hat{x}_{i \mathrm{~g}}$, and $\hat{x}_{i 1}$, we can compute $z_{i}$ by using Eq. 22, because Eq. 22 is independent of the phase density in single-phase regions and, subsequently, we can use the calculated value of $z_{i}$, temperature $T$, and pressure $P$ to obtain the density in single-phase regions. This advantage of the NegSat solution approach does not exist in a variable-set based on compositional space parameterization (Voskov and Tchelepi 2009; Voskov 2010) where $z_{i}$ cannot be computed from known values of $\hat{S}_{\mathrm{g}}$ and $\hat{x}_{i}$, because the molar fraction of the single-phase ( $L$ or $V$ ) should also be known. Consequently, to deal with this issue in the compositional-space-parameterization approach, an additional nonlinear equation with an additional unknown variable ( $L$ or $V$ ) is introduced to the system of equations (Voskov 2010), which adds to computational cost.

\section{Results and Discussion}

We illustrate the non-isothermal NegSat solution approach by way of example to cold mixed $\mathrm{CO}_{2}$-water injection into a geothermal reservoir. Before we describe the numerical model, we first explain possible phase states for $\mathrm{CO}_{2}$-water injection into a geothermal reservoir.

\subsection{Possible Phase States for $\mathrm{CO}_{2}$-Water Injection into a Geothermal Reservoir}

Depending on the overall injected $\mathrm{CO}_{2}$ concentration and based on local thermodynamic equilibrium, the injected $\mathrm{CO}_{2}$ could be in a subcooled or supercritical $\mathrm{CO}_{2}$-rich phase, be completely dissolved in an aqueous (water-rich) phase, or be partly in the subcooled or supercritical $\mathrm{CO}_{2}$-rich phase and partly dissolved in the aqueous phase. Therefore, for cold mixed $\mathrm{CO}_{2}$-water injection into a hot-water reservoir, there are six regions at most in such a flow process: (1) a single-phase region of a subcooled $\mathrm{CO}_{2}$-rich phase, (2) a single-phase region of a supercritical $\mathrm{CO}_{2}$-rich phase, (3) a two-phase region that consists of a gaseous phase with mainly supercritical $\mathrm{CO}_{2}$ and an aqueous phase with mainly water, (4) a two-phase region of a subcooled (liquid) $\mathrm{CO}_{2}$-rich phase and an aqueous phase, (5) a single-phase region of an aqueous (water-rich) phase, and (6) a single-phase region of liquid water. From the practical point of view, the reservoir pressure is usually above the critical $\mathrm{CO}_{2}$ pressure as is the case here. In addition, to have a single-phase region of a subcooled $\mathrm{CO}_{2}$-rich phase or a single-phase region of a supercritical $\mathrm{CO}_{2}$-rich phase, the overall injected $\mathrm{CO}_{2}$ mole fraction must be larger than $99 \%$ (i.e., almost pure $\mathrm{CO}_{2}$ injection) for typical geothermal-reservoir pressures and temperatures. Indeed, subcooled $\mathrm{CO}_{2}$ can be formed near the injection well.

We limit our interest to pressures of practical interest [i.e., hydrostatic pressures at depths of $2 \mathrm{~km}$, which are well above the critical $\mathrm{CO}_{2}$ pressure (73.9 bars)]. If the injection temperature would be below the critical temperature of the mixture, $\mathrm{CO}_{2}$ is subcooled. As the $\mathrm{CO}_{2}$-water mixture moves through the geothermal reservoir, a supercritical $\mathrm{CO}_{2}$ phase can be formed and subsequent dissolution of $\mathrm{CO}_{2}$ in the reservoir water can occur if the temperature increases. In this case, a phase transition between the subcooled and supercritical $\mathrm{CO}_{2}$ takes place. Moreover, depending on the injection condition, one interface between the 
single-phase region and the two-phase region (phase appearance) and one interface between the two-phase region and the hot-liquid-water region (phase disappearance) can exist. It is also possible that there exist only two single-phase regions (viz., the aqueous phase and the pure liquid water phase). This occurs when the $\mathrm{CO}_{2}$ concentration remains below its solubility limit in water for the entire range of temperatures and pressures. The standard solution technique requires that one constantly switches between single-phase and two-phase regions.

\subsection{Numerical Model}

We consider cold mixed $\mathrm{CO}_{2}$-water injection into a geothermal reservoir modeled as a onedimensional (1D) horizontal porous medium initially filled with hot liquid water. The results show the sequence of processes that occur along streamlines. We use the model to understand the sequence of waves that occur along streamlines and to illustrate the non-isothermal NegSat solution approach. The model discussed is a specific example in 1D, with sufficiently high permeabilities to disregard pressure effects due to flow, to facilitate understanding. Although the reservoir pressure is assumed to be constant, the superficial Darcy flux is varying between injection and production wells as one primary unknown variable. In this example, we disregard capillary pressure, gravity, and the diffusion flux of components in phases for reasons of clear illustration. These simplifications improve the fundamental understanding of the application of the non-isothermal NegSat solution approach for flow of mixtures undergoing mass transfer between phases, assuming local thermodynamic equilibrium. However, as it turns out, the solution approach can be easily extended to any non-isothermal two-phase compositional flow problem in $2 \mathrm{D}$ or $3 \mathrm{D}$, including capillary pressure, mass, and thermal diffusion (Salimi et al. 2011). As we are solving the equations numerically, we inevitably introduce numerical diffusion. We take into account longitudinal heat conduction with the porous rock, but again for illustration purposes, we avoid the complication of heat loss to the overburden and underburden. However, heat loss does not affect the derivations of the NegSat model and it can easily be implemented.

Due to high pressures and temperatures, a $\mathrm{CO}_{2}$-water mixture is a non-ideal mixture. Therefore, we use the Peng-Robinson-Stryjek-Vera equation of state with the Modified Huron-Vidal second-order mixing rule that uses the Non-Random Two-Liquid activity coefficient model to determine the phase equilibrium for the non-ideal $\mathrm{CO}_{2}$-water mixture. A volume-shift procedure is applied to obtain an accurate liquid density. Further details about the thermodynamic model can be found in Wahanik et al. (2010). We take into account the density heterogeneity in Darcy's equation and the density variations in the accumulation term of the conservation equations. Moreover, we assume that the permeability and porosity along the geothermal reservoir are constant.

We consider a geothermal reservoir with a length of $1,500 \mathrm{~m}$, a width of $1,500 \mathrm{~m}$, and a height of $100 \mathrm{~m}$. Initially, the reservoir is saturated with hot water. In other words, the initial gas $\left(\mathrm{CO}_{2}\right)$ saturation in the geothermal reservoir is equal to zero. A cold mixture of $\mathrm{CO}_{2}$-water is injected through the entire cross-section of the reservoir from the left side and, subsequently, water and $\mathrm{CO}_{2}$ are produced through the entire cross-section of the reservoir at the opposite side. Table 1 shows the basic input data for the numerical simulations and includes the boundary and initial conditions. The geothermal-reservoir properties correspond to a real geothermal reservoir that is located in the West Netherlands basin, in particular the early Cretaceous Delft sandstone member below Delft (Gilding 2010). The viscosities of the liquid and gaseous phase are approximated by the viscosities of $\mathrm{CO}_{2}$ and water as functions of temperature (Perry and Green 1997), they read 
Table 1 Data used in the numerical simulations

\begin{tabular}{ll}
\hline Reservoir pressure $($ bar $)$ & 200 \\
Initial temperature $(\mathrm{K})$ & 353.15 \\
Injection temperature $(\mathrm{K})$ & 293.15 \\
Injection rate $(\mathrm{m} / \mathrm{s})$ & $2.78 \times 10^{-7}$ \\
Injection flow rate $\left(\mathrm{m}^{3} / \mathrm{s}\right)$ & 0.04167 \\
Rock grain density $\left(\mathrm{kg} / \mathrm{m}^{3}\right)$ & 2,650 \\
Rock specific heat capacity $(\mathrm{J} / \mathrm{kg} / \mathrm{K})$ & 1,000 \\
Total thermal conductivity $(\mathrm{W} / \mathrm{m} / \mathrm{K})$ & 2.1 \\
Porosity & 0.2 \\
Absolute permeability $(\mathrm{mDarcy})$ & 100 \\
Liquid relative permeability & $k_{\mathrm{rl}}=\left(1-S_{\mathrm{g}}\right)^{4}$ \\
Gas relative permeability & $k_{\mathrm{rg}}=S_{\mathrm{g}}^{2}\left(1-\left(1-S_{\mathrm{g}}\right)^{2}\right)$ \\
Residual water saturation & 0 \\
Residual gas saturation & 0 \\
Number of grid cells & 2,000 \\
\hline
\end{tabular}

$$
\begin{aligned}
\mu_{\mathrm{g}}= & 1.6128 \times 10^{-3}-9.0436 \times 10^{-6} T+0.0135 \times 10^{-6} T^{2}-1.9476 \times 10^{-12} T^{3}, \\
\mu_{1}= & -0.0123274+27.1038 T^{-1}-23527.5 T^{-2}+1.01425 \times 10^{7} T^{3} \\
& -2.71342 \times 10^{9} T^{-4}+1.86935 \times 10^{11} T^{-5} .
\end{aligned}
$$

In Eqs. 38 and 39, $T$ is the absolute temperature in Kelvin and the unit of viscosity is $\mathrm{Pa} \cdot \mathrm{s}$. These equations disregard the compositional dependence. To calculate the heat capacity of phase $\alpha$, we assume that the heat capacity of phase $\alpha$ is the sum of the molar heat capacities of its components, i.e., we disregard the energy of mixing. We use the following expressions for the heat capacity of $\mathrm{CO}_{2}$ and water (Perry and Green 1997):

$$
\begin{aligned}
C_{\mathrm{p}, \mathrm{CO}_{2}}= & 45.369+8.6881 \times 10^{-3} T-9.6193 \times 10^{5} T^{-2}, \\
C_{\mathrm{p}, \mathrm{H}_{2} \mathrm{O}}= & 2.7637 \times 10^{2}-2.0901 T+8.125 \times 10^{-3} T^{2} \\
& -0.014116 \times 10^{-3} T^{3}+9.3701 \times 10^{-9} T^{4} .
\end{aligned}
$$

Equations 40 and 41 express the heat capacity in the unit of $(\mathrm{J} / \mathrm{mol} / \mathrm{K})$ and $T$ is the absolute temperature in Kelvin.

For each simulation case, the injection rate is uniform. We discretize the geothermal reservoir into 2,000 grid cells in the $x$ direction. For discretization in space, we use the implicit upwind finite-volume method on the vertex-centered scheme. We tested the convergence $=$ consistency + stability of each of our solutions by comparison of a fine gridded solution to a more coarse gridded solution. For the examples considered by us, the solutions are the same except for numerical dispersion effects. Moreover, we compared some of our solutions to analytical solutions obtained by the method of characteristics to validate the solution.

In the simulations, we vary the overall injected $\mathrm{CO}_{2}$ mole fraction. Based on the possible phase states for $\mathrm{CO}_{2}$-water injection into a geothermal reservoir, we examine seven cases in terms of the overall injected $\mathrm{CO}_{2}$ mole fraction and, consequently, the sequence of phase states and waves. Even if our numerical calculations include thermal conductivity and implicitly numerical dispersion, we adopt for the interpretation the nomenclature (rarefactions, shocks, 
and constant states) from the theory of hyperbolic conservation laws (i.e., in the limit of zero dispersion). A rarefaction is a wave that extends proportional to time. A shock is a sudden transition from one state to another state. A constant state is a region where the dependent variables remain constant. Admittedly, in some cases, we speculated whether a transition was a rarefaction or a shock. Nevertheless, such an interpretation is useful for understanding the various bifurcations (i.e., to distinguish between qualitatively different solutions).

In each of the cases, the type and/or the number of phase states and/or the sequence of waves are different. Moreover, to demonstrate the capability of the non-isothermal NegSat solution approach and the numerical model, we compare the solution of the numerical model with the analytical solution obtained by the wave-curve method (method of characteristics), an extension of the fractional-flow method (Lake 1989), for one example. In all cases, we set a lifetime of 30 years for the geothermal reservoir. If $\mathrm{CO}_{2}$ breakthrough happens before 30 years, the lifetime of the geothermal reservoir is reduced accordingly.

The results are organized as follows. First, we compare the results of the numerical model with the analytical results for one example. Then, we illustrate seven cases by which we discuss the influence of various $\mathrm{CO}_{2}$ injection fractions on the storage/production mechanisms of $\mathrm{CO}_{2}$ and on production of hot water from the geothermal reservoir. Finally, we provide a plot of useful-energy (exergy) extraction versus stored $\mathrm{CO}_{2}$ as a screening method for optimal geothermal recovery and/or maximal storage of $\mathrm{CO}_{2}$.

\subsection{Comparison of the Numerical Model with the Wave-Curve Method}

In order to validate the non-isothermal NegSat solution approach, we compare the numerical solution with the analytical solution obtained with the wave-curve method (an extension of the fractional-flow theory) for one example given in Wahanik et al. (2010). The analytical solution was obtained for the following conditions: the overall injected $\mathrm{CO}_{2}$ mole fraction is 0.29 ; the injection temperature is $298 \mathrm{~K}$; the injection flow rate is $4.22 \times 10^{-6} \mathrm{~m}^{3} /\left(\mathrm{m}^{2} \mathrm{~s}\right)$; and the reservoir pressure and temperature are 100 bars and $358 \mathrm{~K}$, respectively. The gas and liquid relative permeability functions used for obtaining the analytical solution are $k_{\mathrm{rg}}=0.95 S_{\mathrm{g}}^{2}$ and $k_{\mathrm{rl}}=0.5\left(1-S_{\mathrm{g}}\right)^{2}$. We also used the same input data as for the analytical solution to make the comparison as fair as possible. For other cases discussed later, we have used the input data that are shown in Table 1. For details of the wave-curve method, we refer to Wahanik et al. (2010) and Azevedo et al. (2010). Figure 1 compares the numerical NegSat solution to the analytical solution obtained with the wave-curve method. In Fig. 1, the gas saturation versus the temperature of the solution path is plotted. The wave sequence for this example is as follows. There exists a slow Buckley-Leverett shock between the injection state and the intermediate constant state $V_{\mathrm{m}}$, followed by a thermal wave. After that, a Buckley-Leverett rarefaction connects $V_{2}$ to $V_{3}$. Finally, there exists a compositional shock between $V_{3}$ and the initial reservoir condition, traveling with a speed equal to the derivative of the fractional-flow function calculated at $V_{3}$. The end-point speed of the thermal wave coincides with the first point of the Buckley-Leverett rarefaction wave at state $V_{2}$. The speed of state $V_{2}$ is equal to the derivative of the fractional-flow function calculated at $V_{2}$.

Figure 1 illustrates that the numerical solution obtained with the non-isothermal NegSat solution approach captures the Buckley-Leverett shock reasonably accurately. However, there is a slight difference in the thermal wave because of numerical dispersion, and the numerical thermal wave shows a more dispersive wave compared to the analytical thermal wave. Again, Fig. 1 reveals that there is an excellent match between the analytical solution and the numerical solution in the Buckley-Leverett rarefaction wave and the compositional wave. Overall, we conclude that the numerical solution obtained with the non-isothermal 


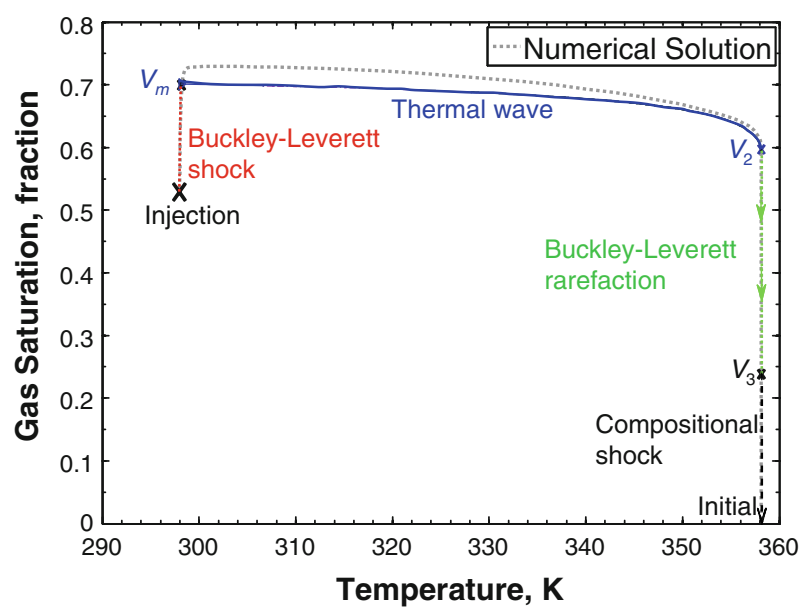

Fig. 1 Solution path for cold $(298 \mathrm{~K})$ mixed $\mathrm{CO}_{2}$-water injection in a single-phase geothermal reservoir (358 $\mathrm{K}$ ) at a pressure of 100 bars; comparison of analytical solutions with numerical solutions. A region of low gradient in the saturation has a high gradient in the temperature

NegSat approach is quantitatively correct and is capable of solving non-isothermal problems that involve phase disappearance and appearance.

\subsection{Case 1}

Case 1 has an overall injected $\mathrm{CO}_{2}$ mole fraction of 0.01 (or about $24.7 \mathrm{~kg}$ of $\mathrm{CO}_{2}$ per ton of water at room conditions). Figure 2 a shows the extended-gas- $\left(\mathrm{CO}_{2}\right)$-saturation profiles at different times for this case. Because the extended gas saturation is below zero for the entire time span considered, Fig. 2a describes a single-phase displacement process in the entire domain. Figure $2 \mathrm{~b}$ shows the temperature profiles at different times for case 1 . By combining the temperature profiles with the extended-gas-saturation profiles, we obtain the corresponding overall- $\mathrm{CO}_{2}$-mole-fraction profiles that are shown in Fig. 2c. Figure $2 \mathrm{c}$ also reveals that $\mathrm{CO}_{2}$ breakthrough has not yet occurred at $t=30$ years.

The solution path of the extended gas saturation in Fig. 2a consists of five parts from the injection side to the initial reservoir condition: the injection constant state, the upstream wave, the constant state, the downstream wave, and the initial constant state at the production side. The upstream wave is a thermal rarefaction wave because it coincides with the thermal wave shown in Fig. 2b. Along the thermal rarefaction wave, the extended gas saturation $\left(\hat{S}_{\mathrm{g}}\right)$, temperature $(T)$, total velocity $(u)$, and $\mathrm{CO}_{2}$ mole fractions in the gaseous and aqueous phase (not shown here) vary, but the overall $\mathrm{CO}_{2}$ mole fraction $\left(z_{\mathrm{CO}_{2}}\right)$ (Fig. $2 \mathrm{c}$ ) is constant. The overall $\mathrm{CO}_{2}$ mole fraction is a saturation-weighted mole fraction. The downstream wave is a compositional wave because it coincides with a change in the overall $\mathrm{CO}_{2}$ mole fraction shown in Fig. 2c; $T$ and $u$ are constant in this wave. The compositional wave is a contact discontinuity along which the overall $\mathrm{CO}_{2}$ mole fraction and the extended gas saturation change, but $T$ and $u$ are constant. Therefore, in this case, there are five regions. Region 1 consists of a single-phase region of an aqueous (water-rich) phase with the injection saturation, temperature, and composition. In region 2, there is a thermal rarefaction wave. In region 2 , the extended gas saturation is still below zero; hence, region 2 is also a single-phase region of an aqueous phase with the overall injected $\mathrm{CO}_{2}$ mole fraction but with varying temperature 
Fig. 2 a Extended-gas-saturation profiles at different times for case 1. Because the extended gas saturation is below zero, it describes a single aqueous phase in the entire domain. The upstream wave is a thermal rarefaction wave; the downstream wave is a compositional wave. In between, there is a constant state. b Temperature profiles at different times for case 1 . There is a thermal rarefaction wave from the injection condition to the initial reservoir condition. c Overall- $\mathrm{CO}_{2}$-mole-fraction profiles at different times for case 1. The profiles consist of a constant state with the injection composition and an initial state with no $\mathrm{CO}_{2}$. In between, there is a compositional wave
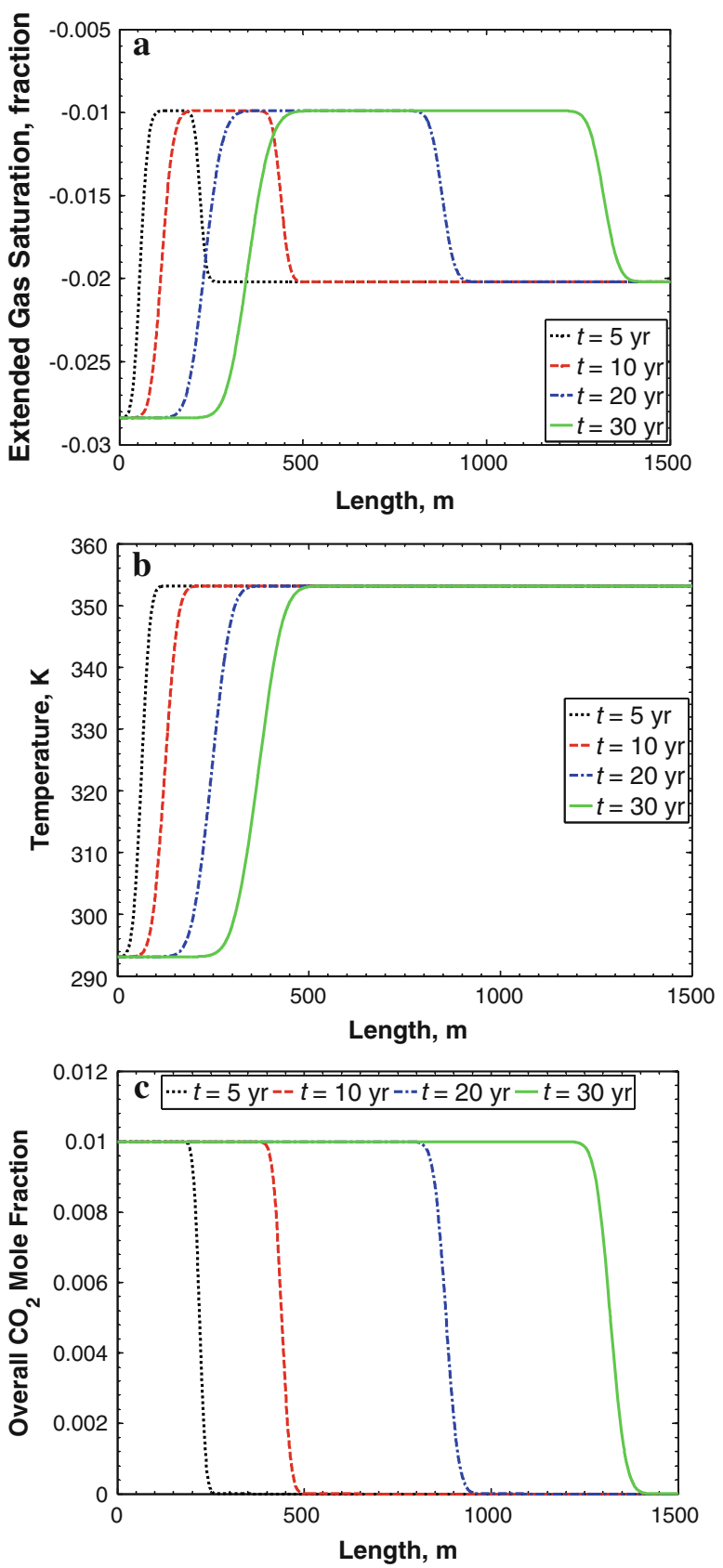

and saturation. Region 3 is a constant state at which $\hat{S}_{\mathrm{g}}, T, u$, and $z_{\mathrm{CO}_{2}}$ are constant. Region 3 is again a single-phase region of an aqueous phase with the injection composition but at the initial temperature. In region 4 , there is a compositional wave; $T$ and $u$ are constant in this wave. Finally, region 5 is a single-phase hot liquid water region at the initial reservoir conditions. 
Figure $2 b$ shows that there is one thermal wave from the injection condition to the initial reservoir condition. Indeed, the regions of low temperature and high temperature are separated by the thermal rarefaction wave (region 2). The temperature dependence of the heat capacities causes some spreading of the wave. Moreover, these waves show an expanding dispersive behavior due to thermal conduction, which has been included in the numerical model.

Figure 2c shows that the overall- $\mathrm{CO}_{2}$-mole-fraction profiles consist of a constant state (regions 1-3) with the injection composition and an initial state with no $\mathrm{CO}_{2}$ (region 5). In between, there is a compositional wave (region 4). This wave runs in front of the thermal wave with a speed that is approximately four times larger than that of the thermal wave.

In the absence of conduction, diffusion, and temperature dependence of heat capacities and phase densities, the speed ratio of the compositional wave to the thermal wave in this single-phase displacement example would be

$$
\frac{v_{\mathrm{c}}}{v_{\mathrm{th}}}=\frac{u}{\varphi} \frac{(1-\varphi)(\rho c)_{\mathrm{s}}+\varphi(\rho c)_{1}}{u(\rho c)_{1}}=\frac{(1-\varphi)(\rho c)_{\mathrm{s}}}{\varphi(\rho c)_{1}}+1 \gg 1,
$$

where $v_{\mathrm{c}}$ and $v_{\text {th }}$ are the speeds of the compositional wave and thermal wave respectively, $\varphi$ is the porosity, $(\rho c)$ is the heat capacity per unit volume, and the subscripts $s$ and $l$ denote the solid and the liquid, respectively. We compute that approximately $v_{\mathrm{c}} / v_{\mathrm{th}}=3.53$.

\subsection{Case 2}

Case 2 has an overall injected $\mathrm{CO}_{2}$ mole fraction of 0.02. As Fig. 3a illustrates, the extended gas saturation is below zero both at the injection side and at the production side, indicating single-phase aqueous regions. In between, we observe a bank, which at early times appears as a peak due to dispersion effects. The bank grows with time, where the extended gas saturation increases from below zero to above zero, indicating a two-phase region. Because the peak grows and has not been yet fully developed until 30 years (the lifetime of the geothermal reservoir), we run this case for longer time. Figure 3 a clearly reveals that the shape of the peak and the sequence of waves change with time. Initially, there is no $\mathrm{CO}_{2}$ peak. It starts to be formed after injection. Figure $3 \mathrm{~b}$ shows the temperature profiles at different times for case 2. Figure $3 \mathrm{c}$ demonstrates the corresponding overall- $\mathrm{CO}_{2}-$ mole-fraction profiles. Moreover, Fig. $3 \mathrm{c}$ reveals that $\mathrm{CO}_{2}$ breakthrough has not yet occurred at $t=30$ years, the lifetime of the geothermal reservoir. Therefore, based on this lifetime, $\mathrm{CO}_{2}$ injection does not limit heat extraction in this case.

The developed solution path of the extended gas saturation shown in Fig. 3a consists of nine parts from the injection side to the initial reservoir condition: the injection constant state, the single-phase thermal rarefaction wave, the evaporation shock, the two-phase thermal rarefaction wave, the condensation shock, the condensation rarefaction wave, the bubble-point constant state, the compositional wave, and the initial constant state at the production side.

Therefore, based on these solution parts, there are seven regions and two shocks in case 2. Region 1 is a single-phase region of an aqueous (water-rich) phase with the injection saturation, temperature, and composition. In region 2, there is a single-phase thermal rarefaction wave. In this wave, the extended gas saturation, temperature, and total velocity change, but the overall $\mathrm{CO}_{2}$ mole fraction is constant and equal to the overall injected $\mathrm{CO}_{2}$ mole fraction. From upstream to downstream in region 2, the extended gas saturation increases because the temperature is higher at the downstream side. However, region 2 is still a single-phase region of an aqueous phase because the extended gas saturation $\hat{S}_{\mathrm{g}}<0$. Regions 2 and 3 are separated by an evaporation shock. Its main feature is that the extended gas saturation increases 
Fig. 3 a The

extended-gas-saturation profiles at different times for case 2 . The extended gas saturation is below zero at the injection side and at the initial condition, indicating single-phase aqueous regions. In between, we observe a peak that grows with time where the extended saturation increases from below zero to above zero, indicating a two-phase region.

b Temperature profiles at different times for case 2 . There is one wave from the injection temperature to the initial reservoir temperature. c Overall- $\mathrm{CO}_{2}$-mole-fraction profiles at different times for case 2. We observe a peak in the overall $\mathrm{CO}_{2}$ mole fraction that coincides with the peak in the extended gas saturation. From the injection side to the production side, the solution path consists of the injection constant state, the evaporation shock, the two-phase thermal rarefaction wave, the condensation shock, the condensation rarefaction wave, the bubble-point constant state, the compositional wave, and the initial constant state at the production side
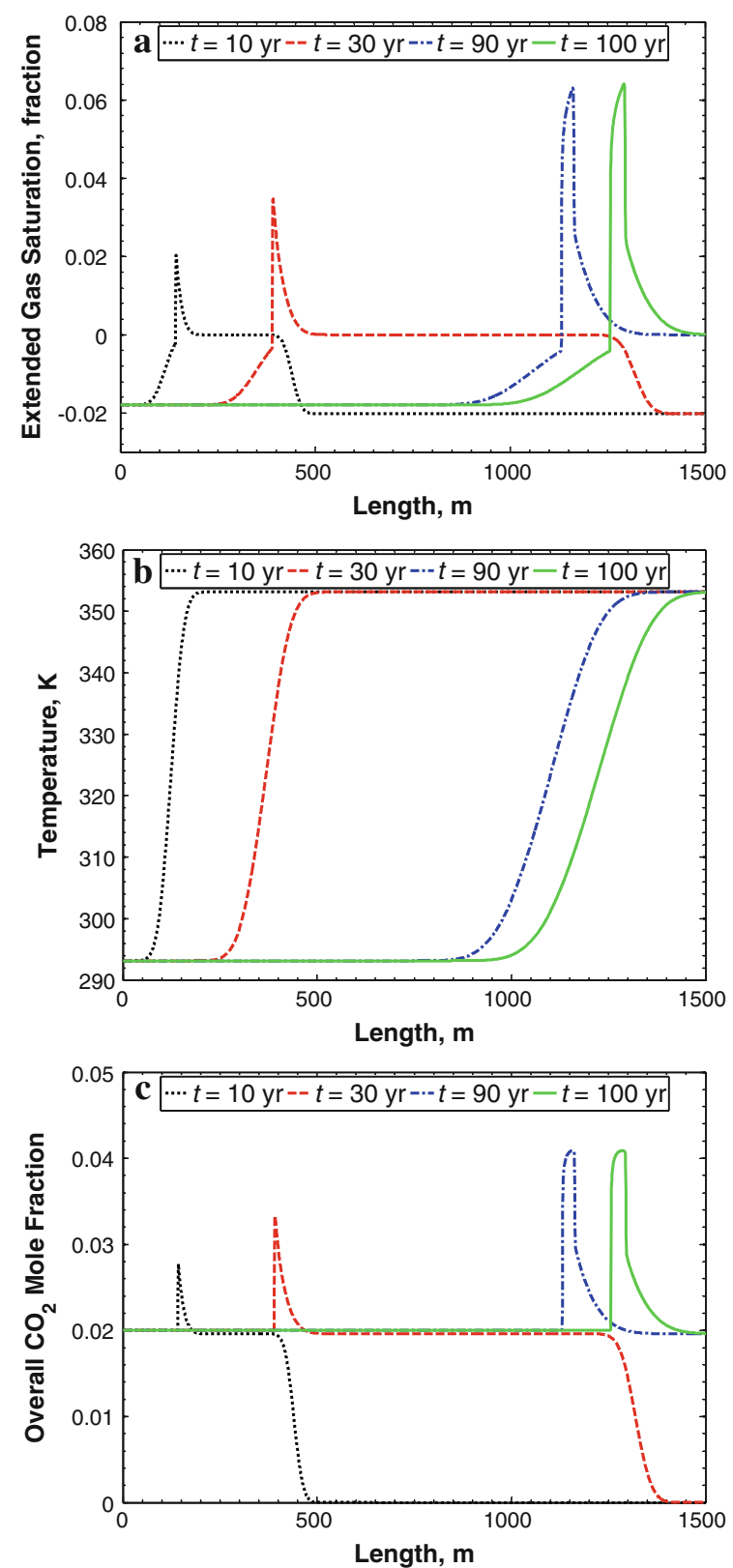

from below zero in the upstream side of the shock to above zero in the downstream side of the shock. The temperature, saturation, composition, and total velocity change across the shock. Indeed, downstream of the evaporation shock, appearance of the gas phase occurs. Therefore, region 3 is a two-phase region that consists of a gas phase with mainly supercritical $\mathrm{CO}_{2}$ and an aqueous phase with mainly water. In region 3, there is a two-phase thermal rarefaction wave and all the quantities change. Regions 3 and 4 are separated by a condensation shock. 
In the condensation shock, the extended gas saturation decreases drastically in the downstream direction. Again, all the quantities change across the condensation shock. In region 4, there is a condensation rarefaction wave, in which all quantities vary. The main feature of the condensation wave is that the extended gas saturation decreases in the downstream direction and reaches zero at the bubble point. In other words, gas-phase disappearance occurs at the downstream side of the condensation rarefaction wave. Moreover, the condensation wave at increases the temperature to its initial value. Because $\hat{S}_{\mathrm{g}}$ is above zero, region 4 is still a two-phase region of a supercritical $\mathrm{CO}_{2}$-rich phase and an aqueous phase. In region 5, there is a constant state at the bubble point (i.e., $\hat{S}_{\mathrm{g}}=0$ ). In the bubble-point constant state, all the quantities are constant. Moreover, the temperature is equal to the initial reservoir temperature and the overall $\mathrm{CO}_{2}$ mole fraction is slightly smaller than the injection concentration. Region 5 consists of a single-phase region of an aqueous (water-rich) phase at the bubble point. In region 6 , there is a compositional wave. In the compositional wave, the extended gas saturation and the overall $\mathrm{CO}_{2}$ mole fraction change, but $T$ and $u$ are constant. Region 6 is also a single-phase region of an aqueous phase. Finally, region 7 contains a single-phase hot-liquid-water region at the initial reservoir conditions.

For the temperature profiles shown in Fig. 3b, the thermal rarefaction wave extending from low-temperature to high-temperature covers regions 2-4.

Figure $3 \mathrm{c}$ illustrates that a peak in the overall $\mathrm{CO}_{2}$ mole fraction coincides with the peak in the extended gas saturation (regions 3 and 4). Downstream of the peak, there exists a constant state (region 5) at which the mole fraction is somewhat lower than the injected $\mathrm{CO}_{2}$ mole fraction. We note that the overall mole fraction is a saturation-weighted mole fraction.

We compare case 1 with case 2 . Both cases are in a single-phase region at the injection side. However, the displacement process remains in single-phase regions for case 1 , whereas the displacement process for case 2 involves single-phase and two-phase regions. Therefore, in case 2, phase appearance and disappearance occur. Furthermore, the overall- $\mathrm{CO}_{2}$-molefraction profiles in case 1 are monotonically decreasing from the injection side toward the production side. However, the behavior of case 2 is different from that of case 1 , because the overall- $\mathrm{CO}_{2}$-mole-fraction profiles show a peak that grows with time. In other words, in case 2, we have accumulated $\mathrm{CO}_{2}$ in the supercritical gas phase. Note that during the entire production and injection time, $\mathrm{CO}_{2}$ has been injected into the reservoir.

\subsection{Case 3}

Case 3 has an overall injected $\mathrm{CO}_{2}$ mole fraction of 0.03 . Figure $4 \mathrm{a}$ shows the extendedgas- $\left(\mathrm{CO}_{2}\right)$-saturation profiles at different times for this case. The extended gas saturation is below zero both at the injection side and at the initial reservoir condition, indicating single-phase aqueous regions. However, in between, we observe a $\mathrm{CO}_{2}$ bank, at which the extended gas saturation is above zero, indicating two-phase regions. Figure $4 \mathrm{~b}$ displays the temperature profiles at different times. Figure $4 \mathrm{c}$ presents the corresponding overall- $\mathrm{CO}_{2}-$ mole-fraction profiles. In addition, Fig. $4 \mathrm{c}$ reveals that $\mathrm{CO}_{2}$ breakthrough has not yet occurred at $t=30$ years, meaning that $\mathrm{CO}_{2}$ injection does not reduce heat extraction in this case.

Figure 4a illustrates that the solution path of the extended gas saturation consists of nine parts from the injection side to the production side: the injection constant state, the singlephase thermal rarefaction wave, the evaporation shock, the two-phase thermal rarefaction wave, the two-phase constant state, the Buckley-Leverett shock, the bubble-point constant state, the compositional wave, and the initial constant state at the production side. Therefore, there are seven regions and two shocks in case 3. Region 1 is a single-phase region of an aqueous phase with the injection saturation, temperature, and composition. In region 2 , there 
Fig. 4 a Extended-gas-saturation profiles at different times for case 3 . The extended gas saturation is below zero at the injection side and at the initial condition, indicating a single-phase aqueous region. However in between, the extended gas saturation is above zero, indicating two-phase regions. b Temperature profiles at different times for case 3 . There is one wave from the injection temperature to the initial reservoir temperature.

c Overall- $\mathrm{CO}_{2}$-mole-fraction profiles at different times for case 3 . We observe a peak in the overall $\mathrm{CO}_{2}$ mole fraction that coincides with the peak in the extended gas saturation. From the injection side to the production side, the solution path consists of the injection constant state, the evaporation shock, the two-phase non-monotonous thermal rarefaction wave, the constant state, the Buckley-Leverett shock, the bubble-point constant state, the compositional wave, and the initial constant state at the production side
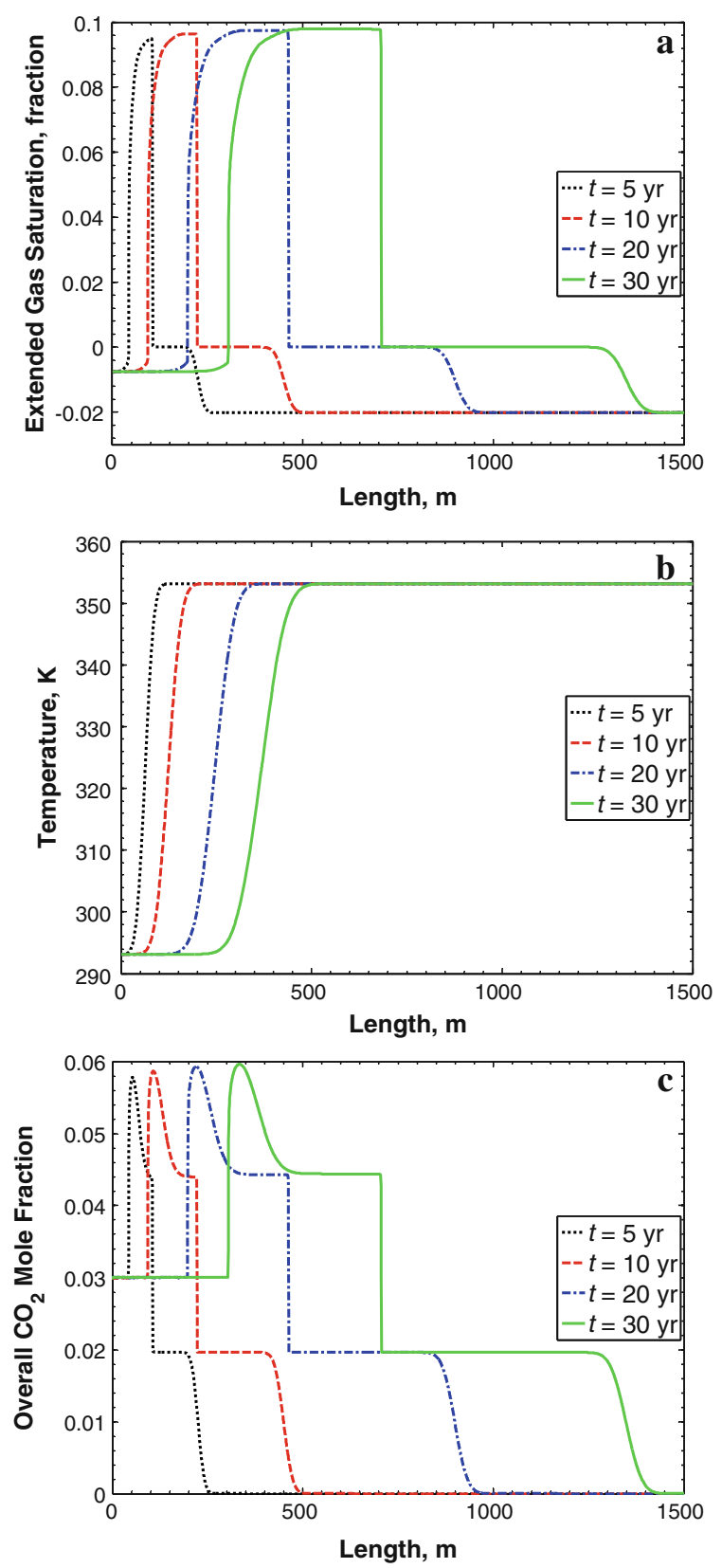

is a single-phase thermal rarefaction wave. We note that the $\mathrm{CO}_{2}$ mole fractions in the gaseous and aqueous phase vary whenever the temperature changes. Regions 2 and 3 are separated by an evaporation shock. Upstream of the evaporation shock, the extended gas saturation is below zero and downstream of the shock, the extended gas saturation is above zero. All the quantities change across this shock. The temperature increase is the reason that the extended gas saturation increases in this evaporation shock. Indeed, gas-phase appearance occurs after 
the evaporation shock. Region 3 is a two-phase region (i.e., $\hat{S}_{\mathrm{g}}>0$ ) with a gas phase with mainly supercritical $\mathrm{CO}_{2}$ and an aqueous phase with mainly water. In region 3 , there is a two-phase thermal rarefaction wave, in which the extended gas saturation, temperature, and $\mathrm{CO}_{2}$ composition vary. In addition, the extended gas saturation increases, meaning that evaporation continues. However, the overall $\mathrm{CO}_{2}$ mole fraction first increases and then decreases. Region 4 is a two-phase constant state at which the extended gas saturation, temperature, total velocity, and overall $\mathrm{CO}_{2}$ mole fraction are constant. Regions 4 and 5 are separated by a Buckley-Leverett shock because only the extended gas saturation and consequently, the overall $\mathrm{CO}_{2}$ mole fraction change across this shock, but $T$ and $u$ are constant. For that reason, the energy conservation equation does not play a role. In a Buckley-Leverett shock, there is no component mass exchange between phases, as opposed to a condensation shock. The Buckley-Leverett shock separates the two-phase region from the single-phase region (at the bubble point). In other words, gas-phase disappearance occurs at the downstream side of the Buckley-Leverett shock. Therefore, region 5 is a single aqueous (water-rich) phase at the bubble point. In region 5, the extended gas saturation, temperature, and $\mathrm{CO}_{2}$ composition are constant. In region 6, there is a compositional wave. Region 6 is an aqueous single-phase region. Finally, region 7 is a single-phase region of hot liquid water at the initial reservoir conditions.

Figure $4 \mathrm{~b}$ demonstrates the same solution path as Fig. $3 b$ for case 2 (i.e., there is one thermal wave from the injection temperature to the initial reservoir temperature).

Figure $4 \mathrm{c}$ upstream shows an evaporation shock that leads to a jump in the overall $\mathrm{CO}_{2}$ mole fraction. After that, we observe a $\mathrm{CO}_{2}$ bank. This $\mathrm{CO}_{2}$ bank coincides with the intermediate rarefaction in the extended gas saturation. The $\mathrm{CO}_{2}$ bank covers region 3 .

\subsection{Case 4}

Case 4 has an overall injected $\mathrm{CO}_{2}$ mole fraction of 0.07 . Figure $5 \mathrm{a}$ reveals that the extended gas saturation is above zero at the injection side, implying that there are two phases at the injection side, as opposed to the previously discussed cases. Figure $5 \mathrm{~b}$ demonstrates the temperature profiles at different times. Figure $5 \mathrm{c}$ displays the overall- $\mathrm{CO}_{2}$-mole-fraction profiles at different times for this case. Figure $5 \mathrm{c}$ also shows that the overall $\mathrm{CO}_{2}$ mole fraction is monotonically decreasing from the injection side toward the production side, as opposed to cases 2 and 3 .

Figure 5a demonstrates that the solution path of the extended gas saturation consists of seven parts from the injection side to the production side: the injection constant state, the thermal rarefaction wave, the two-phase constant state, the Buckley-Leverett shock, the bubble-point constant state, the compositional wave, and the initial constant state at the production side. Therefore, there are six regions and one shock in case 4 . Region 1 contains a two-phase region of a subcooled (liquid) $\mathrm{CO}_{2}$-rich phase and an aqueous phase at the injection conditions. In region 2, there is a thermal rarefaction wave. Along the thermal rarefaction, the extended gas saturation increases (i.e., evaporation occurs) because of the temperature increase, while the overall $\mathrm{CO}_{2}$ mole fraction decreases. Region 2 is also a two-phase region, but a two-phase region that consists of a gaseous phase with mainly supercritical $\mathrm{CO}_{2}$ and an aqueous phase with mainly water. Therefore, indeed, a phase transition from subcooled $\mathrm{CO}_{2}$ to supercritical $\mathrm{CO}_{2}$ takes place at the interface between regions 1 and 2. Region 3 is a two-phase constant state, along which the extended saturation, temperature, and $\mathrm{CO}_{2}$ composition are constant. The overall $\mathrm{CO}_{2}$ mole fraction in region 3 is smaller than the overall injected $\mathrm{CO}_{2}$ mole fraction. Regions 3 and 4 are separated by a Buckley-Leverett shock. The Buckley-Leverett shock separates the two-phase region from the single-phase 
Fig. 5 a Extended-gas-saturation profiles at different times for case 4. At the injection side, the extended gas saturation, $\hat{S}_{\mathrm{g}}>0$, indicates two-phase injection. The Buckley-Leverett shock represents the interface of gas-phase disappearance. b Temperature profiles at different times for case 4 . There is one wave from the injection temperature to the initial reservoir temperature. c Overall- $\mathrm{CO}_{2}$-mole-fraction profiles at different times for case 4 . The profiles are monotonically decreasing from the injection side toward the production side, as opposed to cases 2 and 3. For cases $1-4, \mathrm{CO}_{2}$ breakthrough has not yet occurred at $t=30$ years
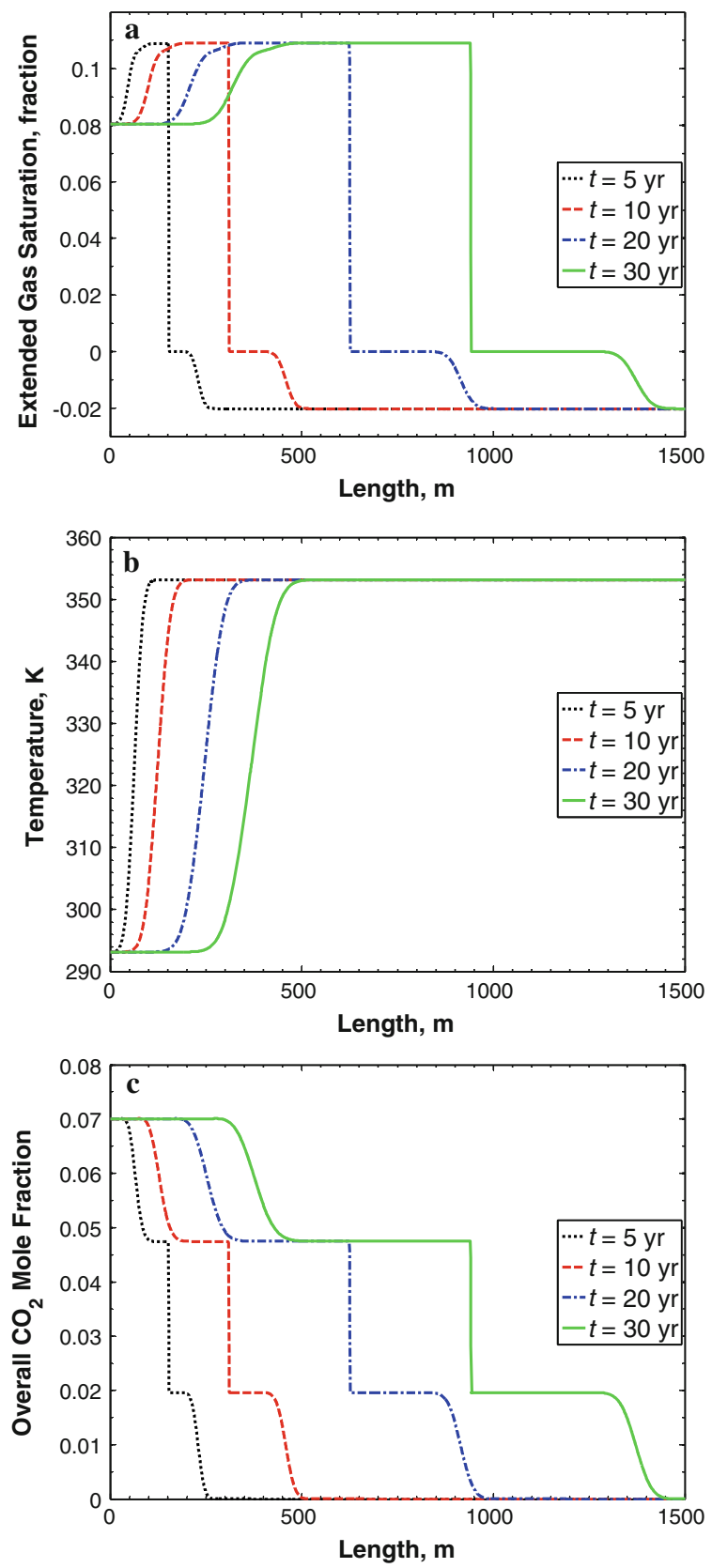

region at the bubble point, because the extended gas saturation is zero in region 4. Region 4 is a bubble-point constant state. In region 5, there is a compositional wave. Region 5 is a single-phase region of an aqueous phase. Finally, region 6 contains a single-phase region of hot liquid water at the initial reservoir conditions. 
Figure $5 \mathrm{~b}$ shows the solution path of the temperature, which is similar to the above cases. A thermal wave connects the low-temperature injection side to the high-temperature initial reservoir condition at the production side. This thermal wave covers region 2 .

Figure 5c illustrates that the composition profiles are monotonically decreasing. This solution behavior is different from the solution behavior of cases 2 and 3 shown in Figs. 3c and $4 c$, respectively.

\subsection{Case 5}

Case 5 has an overall injected $\mathrm{CO}_{2}$ mole fraction of 0.15 . Figure 6 a reveals that the extended gas saturation is above zero at the injection side, implying two-phase conditions at this location. This is similar to case 4; however, the value of the injection extended gas saturation in case 5 is three times higher than that in case 4, because the overall injected $\mathrm{CO}_{2}$ mole fraction is larger in case 5 . Figure $6 \mathrm{~b}$ shows the temperature profiles at different times for case 5. Figure $6 \mathrm{c}$ presents the overall- $\mathrm{CO}_{2}$-mole-fraction profiles at different times for this case. Figure $6 \mathrm{c}$ also demonstrates that $\mathrm{CO}_{2}$ breakthrough has already occurred before 30 years (the lifetime of the geothermal reservoir), meaning that $\mathrm{CO}_{2}$ injection limits heat extraction.

Figure 6a illustrates a completely different solution path with respect to the previous cases. The solution path of the extended gas saturation in case 5 consists of five parts from the injection side to the initial reservoir condition: the injection constant state, the non-monotonous thermal rarefaction wave, the intermediate constant state, the compositional shock, and the initial constant state at the production side. Therefore, there are four regions and one shock in this case. Region 1 is a constant state two-phase region that consists of a subcooled (liquid) $\mathrm{CO}_{2}$-rich phase and an aqueous phase. In region 1, all the dependent variables are constant and equal to the injection values. Subsequently, there is a thermal rarefaction wave in region 2. Comparing this thermal rarefaction wave to the temperature (Fig. 6b), overall- $\mathrm{CO}_{2}$-molefraction (Fig. 6c), and velocity profiles (not shown here), it is plausible that it only concerns one rarefaction wave. In this wave, only the extended gas saturation is non-monotonous (i.e., the extended gas saturation first increases, then decreases, and again increases and attains a value that is slightly higher than its injection value). In region 2 , the temperature, total velocity, and $\mathrm{CO}_{2}$ mole fraction vary. In region $2, T$ and $u$ are higher than in region 1 , but the overall $\mathrm{CO}_{2}$ mole fraction is lower. Because the extended gas saturation is above zero in this region, region 2 is a two-phase region but with a supercritical $\mathrm{CO}_{2}$-rich phase and an aqueous phase. In other words, a phase transition from the subcooled $\mathrm{CO}_{2}$-rich phase to the supercritical $\mathrm{CO}_{2}$-rich phase occurs at the interface between regions 1 and 2 . In region 3, there is a constant state two-phase region of a supercritical $\mathrm{CO}_{2}$-rich phase and an aqueous phase. In this region, the temperature is equal to the initial reservoir temperature, the extended gas saturation is slightly higher than the injection saturation, the total velocity is higher than the injection velocity, and the overall $\mathrm{CO}_{2}$ mole fraction is lower than the overall injected $\mathrm{CO}_{2}$ mole fraction. Downstream of the two-phase constant state, there is a compositional shock, which marks the boundary between regions 3 and 4 . Note that it is not a Buckley-Leverett shock, because its composition changes across the shock. Upstream of the compositional shock, the extended gas saturation is above zero, whereas downstream of the shock, the extended gas saturation is equal to its initial value; it is below zero. Therefore, the compositional shock separates two-phase region 3 from single-phase region 4 . Indeed, gas-phase disappearance takes place at the interface between regions 3 and 4 (i.e., at the downstream side of the compositional shock). The temperature is constant across the shock, but the total velocity, extended gas saturation, and consequently, the overall $\mathrm{CO}_{2}$ mole fraction change. Region 4 is a single-phase region of hot liquid water at the initial reservoir conditions. 
Fig. 6 a Extended-gas-saturation profiles at different times for case 5. At the injection side, there is a constant state involving two phases. Subsequently, there is a non-monotonous thermal rarefaction wave. Next, there is a constant state followed by a compositional shock to the initial condition. The extended gas saturation at the initial condition is again negative, indicating a single-phase aqueous region. b Temperature profiles at different times for case 5 . There is only one thermal wave connecting the low-temperature region at the injection side to the high-temperature region at the production side.

c Overall- $\mathrm{CO}_{2}$-mole-fraction profiles at different times for case 5. At the injection side, there is a constant state with an injected $\mathrm{CO}_{2}$ mole fraction of 0.15 . Then, there is a single rarefaction to an intermediate mole fraction of 0.097 . This is followed by a constant state and a shock to the initial condition
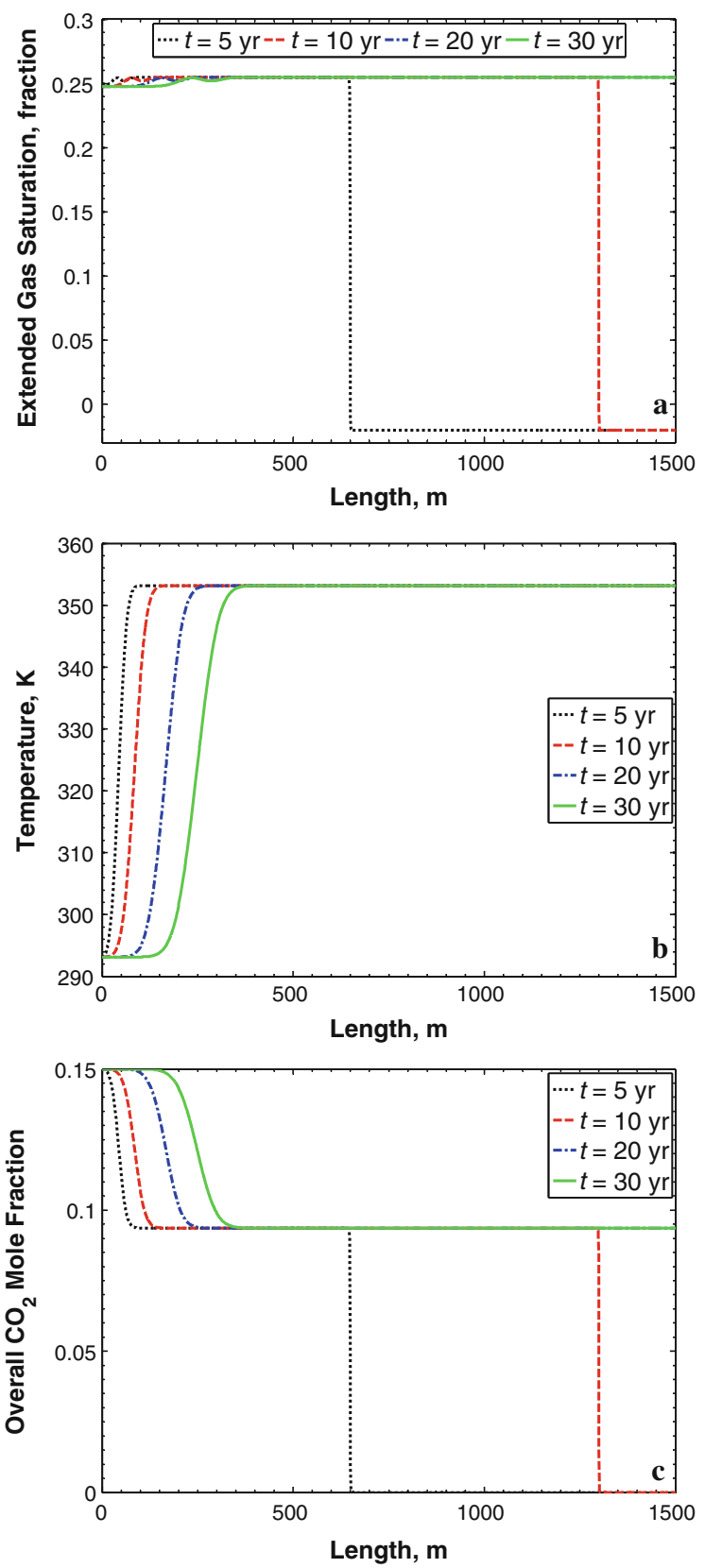

Figure $6 \mathrm{~b}$ shows the same temperature solution path as the above cases. Figure $6 \mathrm{c}$ reveals that the composition profiles are monotonically decreasing.

\subsection{Case 6}

Case 6 has an overall injected $\mathrm{CO}_{2}$ mole fraction of 0.29 . Figure 7 a reveals that the extended gas saturation is above zero at the injection side, implying that there are two phases at the 
Fig. 7 a Extended-gas-saturation profiles at different times for case 6 . The profiles from upstream to downstream show first the injection constant state, the non-monotonous thermal rarefaction, the two-phase constant state, the Buckley-Leverett rarefaction wave at a constant temperature, the compositional shock, and the initial constant state.

b Temperature profiles at different times for case 6 . There is only one thermal wave connecting the low-temperature region at the injection side to the high-temperature region at the production side.

c Overall- $\mathrm{CO}_{2}$-mole-fraction profiles at different times for case 6 . The sequence of waves (solution path) in the overall $\mathrm{CO}_{2}$ mole fraction is the same as that in the extended gas saturation. The overall $\mathrm{CO}_{2}$ mole fraction is monotonically decreasing from the injection side toward the production side
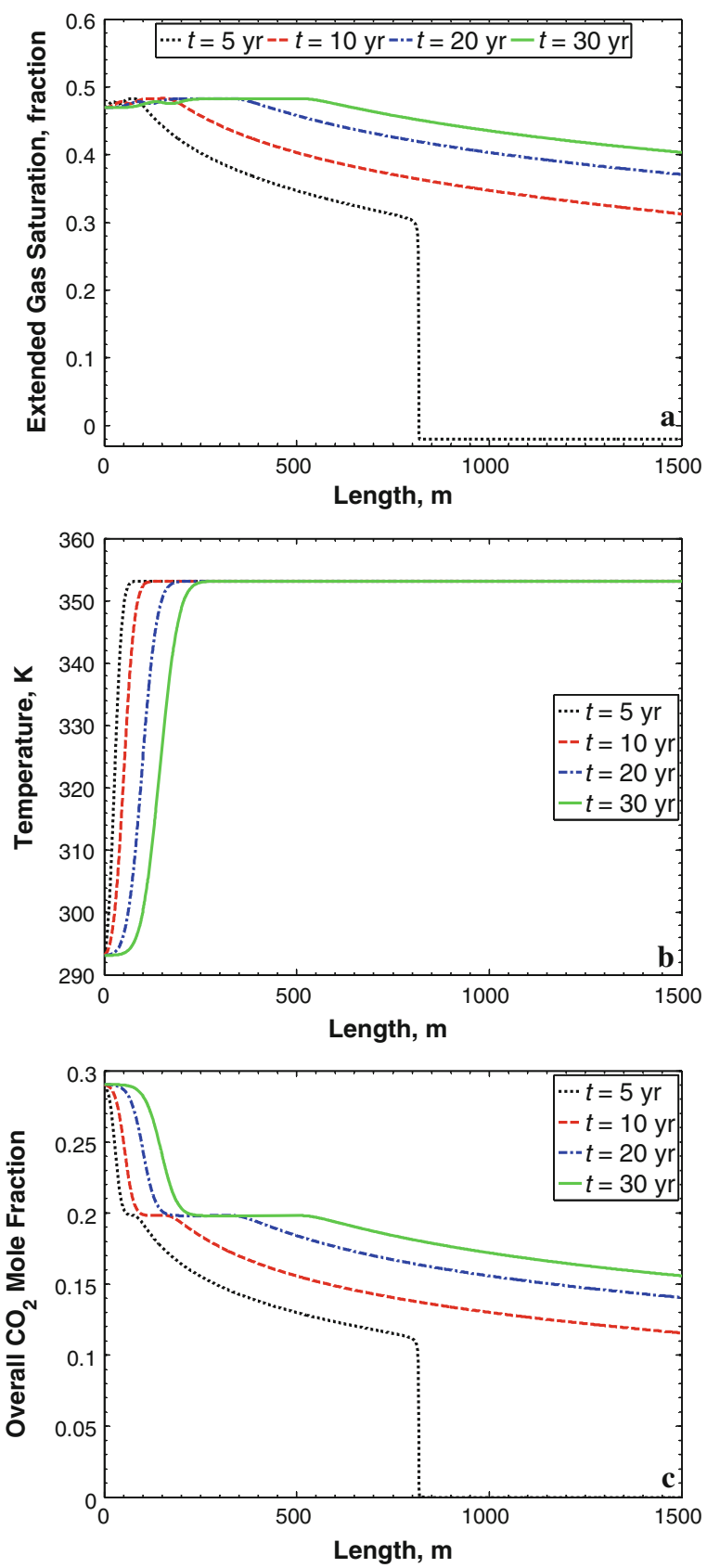

injection side. Figure $7 \mathrm{~b}$ displays the temperature profiles at different times for case 6 . Figure $7 \mathrm{c}$ shows the overall- $\mathrm{CO}_{2}$-mole-fraction profiles at different times for this case. Figure $7 \mathrm{c}$ also illustrates that $\mathrm{CO}_{2}$ breakthrough has already occurred before the end of the lifetime of the geothermal reservoir (30 years). 
Figure 7a describes that the solution path of the extended gas saturation consists of six parts from the injection side to the production side: the constant state at the injection side, the non-monotonous thermal rarefaction wave, the two-phase constant state, the Buckley-Leverett rarefaction wave, the compositional shock, and the initial constant state at the production side. Therefore, there are five regions and one shock in case 6. Region 1 contains a twophase region that consists of a subcooled (liquid) $\mathrm{CO}_{2}$-rich phase and an aqueous phase with the injection temperature, extended gas saturation, and composition. In region 2, there is a thermal rarefaction wave. This thermal rarefaction is non-monotonous in the extendedgas-saturation profiles, whereas it is monotonous in the temperature, total velocity, and the overall- $\mathrm{CO}_{2}$-mole-fraction profiles. In the thermal rarefaction wave, the extended gas saturation first increases, then decreases, again increases and attains a value that is slightly higher than its injection value. In region 2, all the quantities change; the temperature increases and reaches its initial value at the downstream side of the thermal rarefaction wave, the total velocity also increases and attains its highest value. However, the overall $\mathrm{CO}_{2}$ mole fraction monotonically decreases to an intermediate value. Region 2 is also a two-phase region, but a two-phase region of a supercritical $\mathrm{CO}_{2}$-rich phase and an aqueous phase with mainly water. Therefore, a phase transition from subcooled $\mathrm{CO}_{2}$ to supercritical $\mathrm{CO}_{2}$ takes place at the interface between regions 1 and 2. Region 3 is a two-phase constant state, along which the extended saturation, temperature, total velocity, and $\mathrm{CO}_{2}$ composition are constant. The overall $\mathrm{CO}_{2}$ mole fraction in region 3 is smaller than the overall injected $\mathrm{CO}_{2}$ mole fraction. In region 3 , the temperature is equal to its initial value, whereas the total velocity reaches its highest value. In region 4, there is a Buckley-Leverett rarefaction, along which the temperature and total velocity are constant, but the extended gas saturation and consequently the overall $\mathrm{CO}_{2}$ mole fraction monotonically decrease to intermediate values that are smaller than the injection values but are larger than the initial values. Nevertheless, region 4 is a two-phase region of a supercritical $\mathrm{CO}_{2}$-rich phase and an aqueous phase, because the extended gas saturation is above zero $\left(\hat{S}_{\mathrm{g}}>0\right)$. Regions 4 and 5 are separated by a compositional shock. The compositional shock separates the two-phase region from the single-phase region at the initial reservoir conditions, because the extended gas saturation is below zero and is equal to its initial value in region 5. Indeed, gas-phase disappearance takes place at the interface between regions 4 and 5 (i.e., at the downstream side of the compositional shock).

Figure $7 \mathrm{c}$ upstream shows that the sequence of waves in the overall $\mathrm{CO}_{2}$ mole fraction is the same as the sequence in the extended gas saturation.

\subsection{Case 7}

Case 7 has an overall injected $\mathrm{CO}_{2}$ mole fraction of 0.999 (or almost pure $\mathrm{CO}_{2}$ injection). Figure $8 \mathrm{a}$ shows the extended-gas- $\left(\mathrm{CO}_{2}\right)$-saturation profiles at different times for this case. Figure 8 a reveals that the extended gas saturation is slightly above one at the injection side, implying that at the injection side, there is a single-phase region of a subcooled $\mathrm{CO}_{2}$-rich phase, as opposed to all the above cases. Figure $8 \mathrm{~b}$ presents the temperature profiles at different times for case 7 . Figure $8 \mathrm{c}$ illustrates the overall- $\mathrm{CO}_{2}$-mole-fraction profiles at different times for this case.

Figure $8 \mathrm{a}$ demonstrates that the solution path of the extended gas saturation consists of six parts from the injection side to the production side: the injection constant state, the evaporation shock, the thermal rarefaction wave, the Buckley-Leverett rarefaction wave, the compositional shock, and the initial constant state at the production side. Therefore, there are four regions and two shocks in case 7 . Region 1 contains a single-phase region that only consists of a subcooled (liquid) $\mathrm{CO}_{2}$-rich phase at the injection temperature, extended gas 
Fig. 8 a Extended-gas-saturation profiles at different times for case 7. The extended gas saturation is above one, indicating a single-phase subcooled $\mathrm{CO}_{2}$ region. There is a shock to the two-phase region followed by a thermal rarefaction wave. It appears that the constant state is absent and that the thermal rarefaction and Buckley-Leverett rarefaction join at the coincidence point $C$. b Temperature profiles at different times for case 7 . There is only one thermal wave connecting the low-temperature region at the injection side to the high-temperature region at the production side. As the amount of injected $\mathrm{CO}_{2}$ increases, the difference between the speed of the thermal front and the speed of the compositional front becomes larger. c Overall

$\mathrm{CO}_{2}$-mole-fraction profiles at different times for case 7 . The sequence of waves (solution path) in the overall $\mathrm{CO}_{2}$ mole fraction is the same as the sequence in the extended gas saturation. At the injection side, the overall $\mathrm{CO}_{2}$ mole fraction is drastically decreasing to a lower value. The rate of change in the thermal rarefaction wave is higher than that in the Buckley-Leverett rarefaction wave
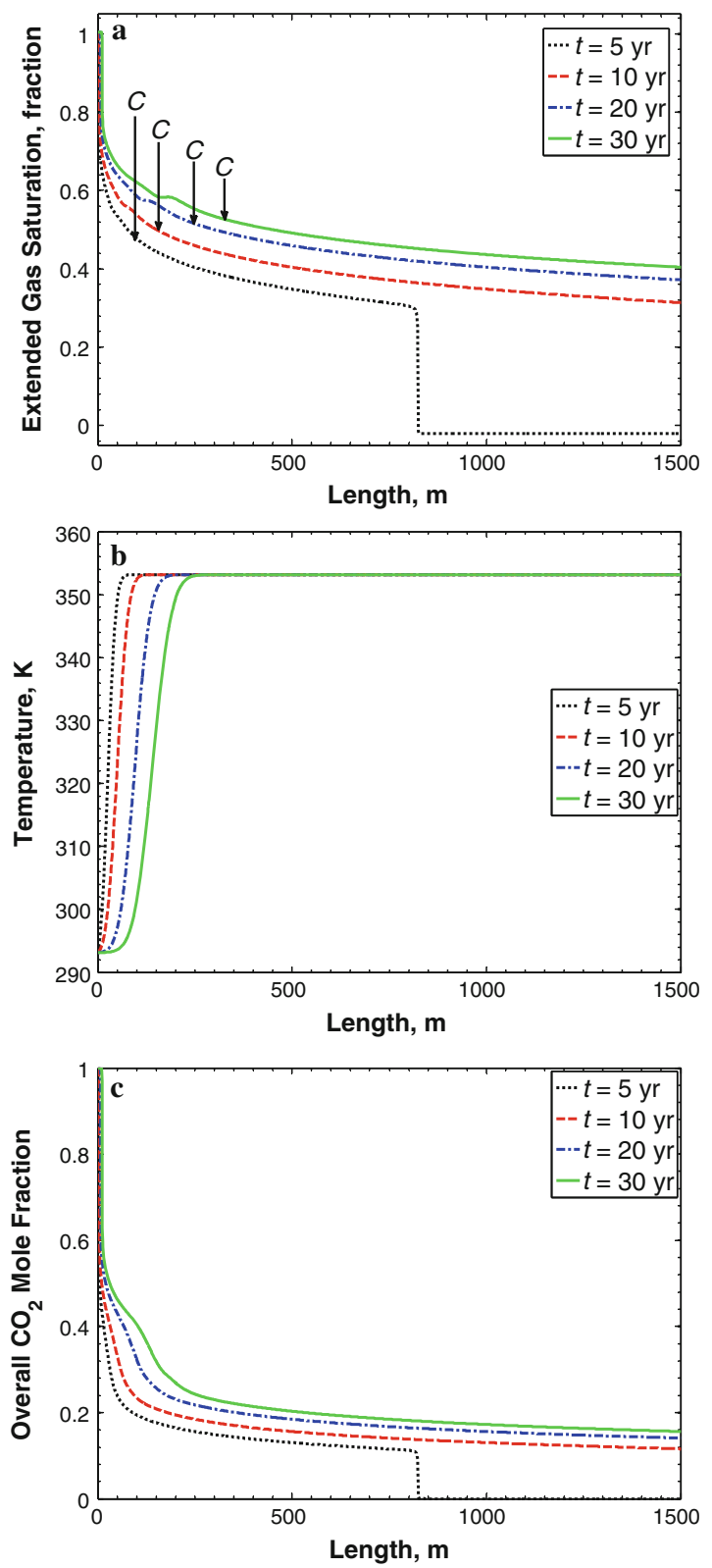

saturation, and composition. Then, there is an evaporation shock, which separates regions 1 and 2. At the evaporation shock, the water at the injection side evaporates. The consequence of this evaporation is that at the upstream of the shock, the extended gas saturation is above one (i.e., a single-phase region), whereas at the downstream side of the evaporation shock, the extended gas saturation is between zero and one (i.e., a two-phase region). Therefore, region 2 is a two-phase region that consists of a supercritical $\mathrm{CO}_{2}$-rich phase and an aqueous phase. Indeed, in this case, (I) a phase transition from subcooled $\mathrm{CO}_{2}$ to supercritical $\mathrm{CO}_{2}$ and (II) 
aqueous-phase appearance take place at the interface between regions 1 and 2 . In region 2, there is a thermal rarefaction wave, along which all the quantities vary. The extended gas saturation and consequently the overall $\mathrm{CO}_{2}$ mole fraction decrease monotonically to intermediate values. The temperature increases to its initial value and total velocity increases to its highest value. As opposed to case 6, it appears that the constant state between the thermal rarefaction wave and the Buckley-Leverett rarefaction wave is absent and that the thermal rarefaction and Buckley-Leverett rarefaction join at point $C$ (see Fig. 8a). At state $C$, the two characteristic speeds coincide. Hence, in region 3, there is a Buckley-Leverett rarefaction, along which the temperature and total velocity are constant, but the extended gas saturation and consequently the overall $\mathrm{CO}_{2}$ mole fraction monotonically decrease to lower intermediate values that are smaller than the injection values but are larger than the initial values. Still, region 3 is a two-phase region of a supercritical $\mathrm{CO}_{2}$-rich phase and an aqueous phase, because the extended gas saturation is above zero $\left(\hat{S}_{\mathrm{g}}>0\right)$. Regions 3 and 4 are separated by a compositional shock. The compositional shock separates the two-phase region from the single-phase region at the initial conditions, because the extended gas saturation is below zero and is equal to its initial value in region 4 . Region 4 contains a single-phase region of hot liquid water at the initial reservoir conditions.

Figure $8 \mathrm{~b}$ shows that there is one thermal wave from the injection temperature to the initial reservoir temperature.

Figure $8 \mathrm{c}$ demonstrates that the sequence of waves in the overall $\mathrm{CO}_{2}$ mole fraction is the same as the sequence in the extended gas saturation.

\subsection{Exergy Recovery Versus $\mathrm{CO}_{2}$ Storage}

Figure 9 plots the useful energy (exergy, see De Swaan Arons et al. 2004) at the end of the process (heat extraction) versus the maximum amount of stored $\mathrm{CO}_{2}$ for various overall injected $\mathrm{CO}_{2}$ mole fractions. These are the salient characteristics of mixed $\mathrm{CO}_{2}$-water injection into a geothermal reservoir. For all cases, the initial reservoir conditions, total injection rate, and injection temperature are the same, while the overall injected $\mathrm{CO}_{2}$ mole fraction is different for each case. Along the $A B C D$ curve, the overall injected $\mathrm{CO}_{2}$ mole fraction increases. At point $\mathrm{A}$, with no added $\mathrm{CO}_{2}$, the criterion to end the project is at cold-water breakthrough. If a tiny amount of $\mathrm{CO}_{2}$ is added, the criterion to end the project is when $\mathrm{CO}_{2}$, dissolved in the aqueous phase, breaks through.

As Fig. 9 reveals, we distinguish six regions in terms of useful energy/stored $\mathrm{CO}_{2}$ : region 1 point $A$ (no $\mathrm{CO}_{2}$ ), region 2 from case 1 to point $B$, region 3 from point $B$ to point $C$, region 4 from point $C$ to case 5 , region 5 from case 5 to point $D$, and region 6 from point $D$ to case 7. Point $A$ corresponds to cold-water injection (i.e., no $\mathrm{CO}_{2}$ ); as a result, it is located on the $y$-axis. Consequently, there is a discontinuous change in terms of heat extraction (useful energy) from $3590 \mathrm{TJ}$ to $1160 \mathrm{TJ}\left(1 T=10^{12}\right)$. This corresponds approximately to the ratio 3.53 calculated using Eq. 41. Figure 9 shows that in region 2 from case 1 to point $B$, the useful energy remains constant, while the stored $\mathrm{CO}_{2}$ increases. In region 2 , the $\mathrm{CO}_{2}$ concentration is low. When the concentration is still low, the compositional wave moves at

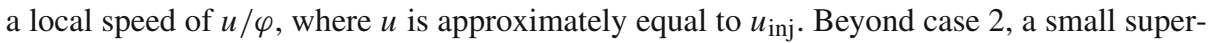
critical $\mathrm{CO}_{2}$-rich phase is formed, but the compositional wave at a local speed of $u / \varphi$ is still the fastest (leading) wave. Hence, $\mathrm{CO}_{2}$ breakthrough is connected to breakthrough of this wave. The total velocity $u$ in case 2 becomes higher than that in case 1 , but the increase is around $0.45 \%$, and consequently, the time of $\mathrm{CO}_{2}$ breakthrough does not decrease significantly. The increase in the total velocity from case 1 to point $B$ is $5.06 \%$. Hence, we observe a change in storage capacity, but the energy extraction that mainly depends on the time of 


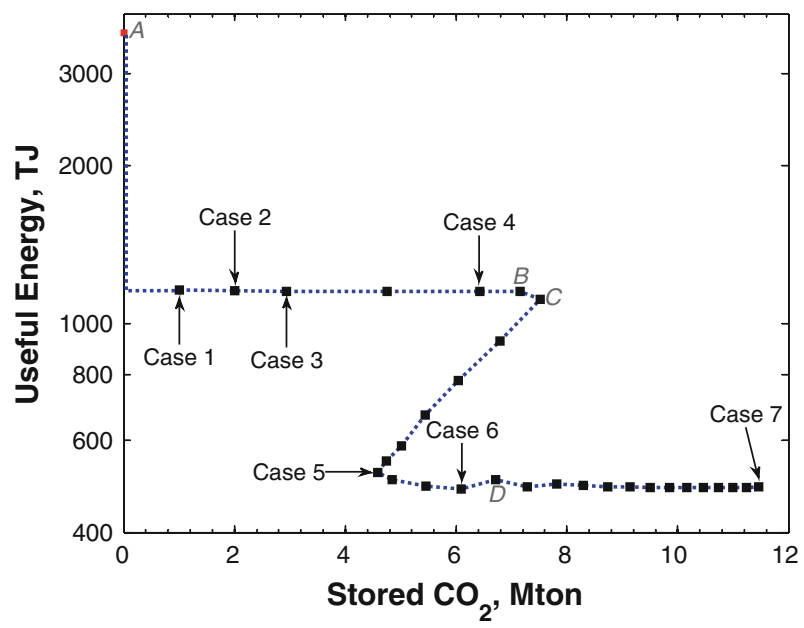

Fig. 9 Useful-energy (exergy) extraction versus stored $\mathrm{CO}_{2}$ at $\mathrm{CO}_{2}$ breakthrough either in the aqueous or in the gaseous phase. The trend from left to right represents an increase in the injected $\mathrm{CO}_{2}$ mole fraction. The $y$-axis is on a $\log$ base 10 scale

$\mathrm{CO}_{2}$ breakthrough remains more or less the same. From point $B$ to point $C$ (region 3 ), the useful-energy/stored- $\mathrm{CO}_{2}$ behavior changes and a transition occurs. The reason is that the sequence of waves changes from point $B$ to point $C$ (cf. Figs. 5c and 6c). At still higher $\mathrm{CO}_{2}$ injection, the shock, the bubble-point constant state, and the compositional wave are overtaken by a compositional shock, which moves much faster than the compositional wave in region 2. From point $C$ to case 5, the sequence of waves remains the same. Therefore, in region 4 , the time of breakthrough gradually decreases and both the possible amount of stored $\mathrm{CO}_{2}$ and the useful energy decrease. After case 5, again, the sequence of waves changes and a Buckley Leverett rarefaction is connected to the compositional shock. Consequently, the useful-energy/stored- $\mathrm{CO}_{2}$ plot changes character. For that reason, the breakthrough time of the compositional shock decreases by a minor amount (13\%) and we see a slight decrease in the useful energy in region 5. However, there is an increase in storage capacity due to the higher $\mathrm{CO}_{2}$ concentrations, which amply compensates earlier breakthrough.

The behavior again changes at point $D$, where the two-phase constant state between the thermal rarefaction and the Buckley-Leverett rarefaction disappears and the produced useful energy slightly increases. From point $D$ onwards until the last point, case 7 , the sequence of waves and the speed of the compositional shock remain the same. Therefore, the energy recovery remains constant, but due to the enhanced injection concentration, the amount of stored $\mathrm{CO}_{2}$ increases. In case 7 (almost pure $\mathrm{CO}_{2}$ ) even though the sequence of the waves changes, but the speed of the compositional shock (fastest wave) remains the same as in the previous regions (from point $D$ to a point just before case 7 ), and the energy recovery remains the same with increased $\mathrm{CO}_{2}$ storage capacity.

In summary, we have a choice of optimal energy recovery of $1160 \mathrm{TJ}$ with a limited maximal storage of 7.1 Mtons $\left(1 \mathrm{M}=10^{6}\right)$ or a lower energy recovery of $490 \mathrm{TJ}$ with a maximal storage of 11.5 Mtons.

The regions discussed above, can be changed by gravity effects, heterogeneity, pressure changes, well outlays, non-uniform injection rates, and salinity; however, the example discussed in this paper shows the basic principles. 


\section{Conclusions}

- It is possible to extend the isothermal NegSat solution approach to non-isothermal conditions. Therefore, it is possible to simulate cold $\mathrm{CO}_{2}$-water injection into a geothermal reservoir, without using switches as in the standard solution technique.

- There is good agreement between the analytical solution obtained with the wave-curve method and the numerical solution obtained with the NegSat approach.

- Increasing the amount of $\mathrm{CO}_{2}$ in the injection mixture leads to six bifurcation points at which the character of the solution changes.

- At low $\mathrm{CO}_{2}$ injection concentrations (mole fraction below $1 \%$, e.g., case 1 ), there is only a single-phase aqueous region. At a mole fraction larger than $1 \%$ and smaller than $3 \%$, (case 2), a gaseous $\mathrm{CO}_{2}$ bank develops.

- At somewhat higher concentrations (case 3), the development of the $\mathrm{CO}_{2}$ bank is different from that in case 2. At concentrations larger than $4 \%$ and below $9 \%$ (case 4 ), there is a two-phase region at the injection side.

- For concentrations above 9\% and below 16\% (case 5), the solution is completely different and includes a non-monotonous rarefaction wave of the extended-gas-saturation profile. At concentrations between $16 \%$ and $35 \%$ (case 6), a Buckley-Leverett wave downstream is separated from a thermal wave with a constant state in between.

- At almost pure $\mathrm{CO}_{2}(99 \%)$ injection (case 7), the constant state between the thermal wave and the Buckley-Leverett wave disappears, meaning that there is a joining point between the two rarefactions.

- In all of the cases, there is a compositional wave that runs ahead of the thermal wave. This limits the period of simultaneous $\mathrm{CO}_{2}$ storage and heat extraction to the end of the project.

- For a field case, the calculations show that $\mathrm{CO}_{2}$ breakthrough may occur after 30 years at overall injected $\mathrm{CO}_{2}$ mole fractions less than 0.07 . Note that effects, such as gravity and salinity have not been included.

- A plot of the useful energy versus the $\mathrm{CO}_{2}$ storage capacity shows a $Z$-shape. We apply it to an example field of $1500 \times 1500 \times 100 \mathrm{~m}^{3}$, with a porosity of $20 \%$. The top horizontal part represents the high exergy $(1160 \mathrm{TJ})$ branch with a maximum of 7.1 Mtons stored $\mathrm{CO}_{2}$. The bottom horizontal part shows the low exergy (490 TJ) branch with a maximum $\mathrm{CO}_{2}$ storage capacity of $11.5 \mathrm{Mtons}$. These data exclude heat gain, heterogeneity, gravity, etc., and thus show only an order of magnitude.

Acknowledgments The work has been performed within the framework of the Dutch National $\mathrm{CO}_{2}$-storage program CATO-2, work-package 3.5. We thank Ali Akbar Eftekhari for providing us with a thermodynamic model. We acknowledge Helmut Wahanik for sending us the analytical solution. We also thank Dan Marchesin for useful discussions.

\section{References}

Abadpour, A., Panfilov, M.: Method of negative saturations for modeling two-phase compositional flow with oversaturated zones. Transp. Porous Media 79(2), 197-214 (2009)

Azevedo, A.V., de Souza, A.J., Furtado, F., Marchesin, D., Plohr, B.: The solution by the wave curve method of three-phase flow in virgin reservoirs. Transp. Porous Media 83(1), 99-125 (2010)

Ben-Omran, A.M., Green, D.W.: A two-dimensional, two-phase compositional model which uses a moving point method. Paper SPE 7415 presented at the SPE annual fall technical conference and exhibition, Houston, TX, 1-3 October 1978 
Bonnerot, R.P. Jamet: A conservative finite element method for one-dimensional Stefan problems with appearing and disappearing phases. J. Comput. Phys. 41(2), 357-388 (1981)

Bruining, J., Marchesin, D.: Maximal oil recovery by simultaneous condensation of alkane and steam. Phys. Rev. E Stat. Nonlinear Soft Matter Phys. 75(3), art. no. 036312 (2007)

Bruining, J.,Van Duijn, C.J.: Traveling waves in a finite condensation rate model for steam injection. Comput. Geosci. 10(4), 373-387 (2006)

Chen, Z., Huan, G., Ma, Y.: Computational Methods for Multiphase Flows in Porous Media. Society for Industrial and Applied Mathematics, Philadelphia (2006)

De Swaan Arons, J., Van der Kooi, H., Sankaranarayanan, K.: Efficiency and Sustainability in the Energy and Chemical Industries, ISBN 0824708458. Marcel Dekker Inc., New York (2004)

Gilding, D.T.: Heterogeneity determination of the Delft subsurface for heat flow modelling. MS thesis, Delft University of Technology, Delft, The Netherlands (2010)

Lake, L.W.: Enhanced Oil Recovery. Prentice Hall, Englewood Cliffs (1989)

Michelsen, M.L.: The isothermal flash problem: Part I. Stability. Fluid Phase Equilib. 9(1), 1-19 (1982a)

Michelsen, M.L.: The isothermal flash problem: Part II. Phase-split calculation. Fluid Phase Equilib. 9(1), 21-40 (1982b)

Panfilov, M., Rasoulzadeh, M.: Interfaces of phase transition and disappearance and method of negative saturation for compositional flow with diffusion and capillarity in porous media. Transp. Porous Media 83(1), 73-98 (2010)

Perry, R.H., Green, D.W.: Perry's Chemical Engineers' Handbook 7th edn. McGraw-Hill, New York (1997)

Pruess, K.: Numerical simulation of $\mathrm{CO}_{2}$ leakage from a geologic disposal reservoir, including transitions from super- to subcritical conditions, and boiling of liquid $\mathrm{CO}_{2}$. Soc. Petrol. Eng. J. 9(2), 237-248 (2004)

Salimi, H., Wolf, K-H., Bruining, J.: Negative saturation approach for compositional flow simulations of mixed $\mathrm{CO}_{2}$-water injection into geothermal reservoirs including phase transition and disappearance. Paper SPE 142924 presented at the SPE EUROPEC/EAGE annual conference and exhibition, Vienna, Austria, 23-26 May 2011

Voskov, D.V.: A new variable-set for nonlinear flow simulation based on compositional space parameterization. Paper A015 presented at the 12th European conference on the mathematics of oil recovery (ECMOR), Oxford, UK, 6-9 September 2010

Voskov, D., Tchelepi, H.: Compositional space parameterization: multi-contact miscible displacements and extension to multiple phases. Soc. Petrol. Eng. J. 14(3), 441-449 (2009)

Wahanik, H., Eftekhari, A.A., Bruining, J., Marchesin, D.: Analytical solutions for mixed $\mathrm{CO}_{2}$-water injection in geothermal reservoirs. Paper SPE 138154 presented at the Canadian unconventional resources and international petroleum conference held in Calgary, Alberta, Canada, 19-21 October 2010

Whitson, C.H., Michelsen, M.L.: The negative flash. Fluid Phase Equilib. 53(C), 51-71 (1989)

Zhu, J., Jessen, K., Orr, F.M., Jr.: Analytical solution for gas/oil displacement with temperature variation. Paper SPE 89432 presented at the SPE/DOE symposium on improved oil recovery, Tulsa, Oklahoma, 17-21 April (2004) 